\title{
Efficacy of broflanilide (VECTRONTM T500) a new meta-diamide insecticide, for indoor residual spraying against pyrethroid-resistant malaria vectors.
}

Corine Ngufor ( $\square$ corine.ngufor@lshtm.ac.uk)

London School of Hygiene \& Tropical Medicine

Renaud Govoetchan

London School of Hygiene \& Tropical Medicine

Augustin Fongnikin

Centre de Recherche Entomologiques de Cotonou

\section{Estelle Vigninou}

Centre de Recherche Entomologiques de Cotonou

Thomas Syme

London School of Hygiene \& Tropical Medicine

\section{Martin Akogbeto}

Centre de Recherche Entomologiques de Cotonou

\section{Mark Rowland}

London School of Hygiene \& Tropical Medicine

\section{Research Article}

Keywords: broflanilide (VECTRONTM T500), insecticide, pyrethroid-resistant malaria vectors

Posted Date: December 10th, 2020

DOl: https://doi.org/10.21203/rs.3.rs-119606/v1

License: (a) (i) This work is licensed under a Creative Commons Attribution 4.0 International License. Read Full License

Version of Record: A version of this preprint was published at Scientific Reports on April 12th, 2021. See the published version at https://doi.org/10.1038/s41598-021-86935-3. 
1 Efficacy of broflanilide (VECTRON ${ }^{\mathrm{TM}}$ T500), a new meta-diamide insecticide,

2 for indoor residual spraying against pyrethroid-resistant malaria vectors.

3 Authors: Corine Ngufor ${ }^{1,2,3^{*}}$ Renaud Govoetchan ${ }^{1,3}$, Augustin Fongnikin ${ }^{2,3}$, Estelle Vigninou², Thomas

4 Syme ${ }^{1,3}$, Martin Akogbeto ${ }^{2,3}$, Mark Rowland ${ }^{1,3}$

5

6 Affiliations:

$7 \quad{ }^{1}$ London School of Hygiene and Tropical Medicine (LSHTM), London, UK

$8 \quad{ }^{2}$ Centre de Recherche Entomologiques de Cotonou (CREC), Benin

${ }^{3}$ Panafrican Malaria Vector Research Consortium (PAMVERC), Benin

10

*corresponding author: Corine Ngufor

12

Email: corine.ngufor@Ishtm.ac.uk

13 
Abstract

2

The rotational use of insecticides with different modes of action for indoor residual spraying (IRS) is recommended for improving malaria vector control and managing insecticide resistance. Insecticides with new chemistries are urgently needed. Broflanilide is a newly discovered insecticide under consideration. We investigated the efficacy of a wettable powder (WP) formulation of broflanilide (VECTRON ${ }^{\mathrm{TM}}$ T500) for IRS on mud and cement wall substrates in laboratory and experimental hut studies against pyrethroid-resistant malaria vectors in Benin, in comparison with pirimiphos-methyl CS (Actellic ${ }^{\circledR}$ 300CS). There was no evidence of cross-resistance to pyrethroids and broflanilide in CDC bottle bioassays. In laboratory cone bioassays, broflanilide WP treated substrates killed $>80 \%$ susceptible and pyrethroid-resistant An. gambiae s/ for 6-14 months. At application rates of $100 \mathrm{mg} / \mathrm{m}^{2}$ and $150 \mathrm{mg} / \mathrm{m}^{2}$, mortality of wild pyrethroid-resistant $A n$. gambiae s/ entering experimental huts in Covè, Benin treated with VECTRON ${ }^{\mathrm{TM}}$ T500 was similar to pirimiphos-methyl CS (57\%-66\% vs. 56\%, $P>0.05)$. Throughout the 6-month hut trial, monthly wall cone bioassay mortality on VECTRON ${ }^{\mathrm{TM}} T 500$ treated hut walls remained $>80 \%$. IRS with broflanilide shows potential to significantly improve the control of malaria transmitted by pyrethroid-resistant mosquito vectors and could thus be a crucial addition to the current portfolio of IRS insecticides.

(1)

(1)

(1) 
Indoor residual spraying (IRS) has historically been shown to be a powerful malaria control intervention ${ }^{1}$. When applied correctly, IRS can quickly reduce malaria transmission by reducing adult mosquito vector density and longevity. It involves the application of a residual insecticide formulation to potential resting surfaces for malaria vectors such as internal walls, eaves and ceilings of houses, giving opportunity for vector mosquitoes to contact the insecticide and be killed in the process. IRS contributed substantially to the success of the malaria eradication campaign of the 1950s and 60s which resulted in the elimination of malaria from Europe and several countries in Asia and the Caribbean ${ }^{2}$. The recent reductions in malaria morbidity and mortality observed in endemic countries in Africa and Asia over the last two decades has also been partly attributed to a significant increase in coverage with IRS ${ }^{3-5}$.

The efficacy of IRS for malaria control is unfortunately threatened by widespread resistance in malaria vectors to the rather limited collection of insecticides approved for public health use ${ }^{6}$. Pyrethroid resistance is now established across Africa and is increasing substantially in intensity the more they are used, making this previously ideal class of vector control insecticide almost unusable for IRS. Resistance to carbamates and organophosphates, which were for many years the only alternative IRS insecticide classes to pyrethroids 5,7 is also increasing rapidly in malaria vector populations in Africa ${ }^{6,8-}$ ${ }^{11}$. To mitigate the impact of insecticide resistance on malaria control, vector control programmes are encouraged to implement a rotational application of insecticides for IRS, alternating between insecticides with different modes of action ${ }^{12}$. The use of rotations for insecticide resistance management relies on the concept that removing selection pressure for a given insecticide by switching between different modes of action, will result in resistance declining over time. An IRS rotation plan which will effectively reduce selection pressure for existing insecticide resistance genes and prevent the development of further resistance will, however, require a more diversified portfolio of IRS insecticides with more novel modes of action than what is currently available ${ }^{13}$. This is driving the development of a new generation of IRS insecticide formulations containing new chemistries which can provide improved and prolonged control of insecticide-resistant malaria vector populations 14-16.

Broflanilide is a novel insecticide discovered by Mitsui Chemicals Agro, Inc ${ }^{17}$ which has been formulated as a wettable powder for IRS. It has a unique chemical structure characterized as a metadiamide which acts as a non-competitive antagonist of the $\gamma$-aminobutyric acid (GABA) receptor of chloride channels of the insect inhibitory nervous system ${ }^{18}$. Broflanilide was classified by the 
(IRAC Group 30), causing hyperexcitation and convulsion in insects ${ }^{19}$. Its mode of action is distinct from that of conventional non-competitive antagonists (NCAs) of the GABA-gated chloride channel, such as picrotoxinin, dieldrin, fipronil, lindane and $\alpha$-endosulfan ${ }^{20}$. There is no known cross-resistance between broflanilide and current public health insecticides. The active metabolite exhibits high selectivity for the insect RDL GABA receptor compared to the mammalian receptors ${ }^{18}$ but exhibits no cross-resistance to dieldrin. Broflanilide has demonstrated excellent insecticidal activity against many insect species including Lepidopteran and Coleopteran pests and Thysanopteran pests ${ }^{17}$ and has also shown low acute toxicity against non-target aquatic organisms ${ }^{21}$ demonstrating high potential for public health and agricultural use.

In this study, we investigated the potential of VECTRON ${ }^{\mathrm{TM}}$ T500, a wettable powder (WP) formulation of broflanilide (broflanilide WP), for indoor residual spraying against mosquito vectors of malaria. The insecticide was assessed for its efficacy and residual activity on local IRS wall substrates in a series of WHO phase I laboratory bioassays with susceptible Anopheles gambiae s.s. and resistant strains of $A n$. gambiae s/ and in WHO phase II experimental huts studies against wild free-flying pyrethroid-resistant of An. gambiae sl in southern Benin, West Africa.

\section{Results}

\section{WHO Phase I laboratory bioassays}

Following WHO guidelines ${ }^{22}$, laboratory bioassays were performed to investigate possible crossresistance to broflanilide and pyrethroid-resistance mechanisms in CDC bottle bioassays using technical grade insecticide and to identify an effective dose of broflanilide WP (VECTRON ${ }^{\mathrm{TM}}$ T500), for IRS using WHO cone bioassays. Cone bioassays were also performed to investigate the residual efficacy of broflanilide WP on cement and mud block substrates. The bioassays were conducted using laboratory maintained mosquitoes of the susceptible An. gambiae ss Kisumu strain and the pyrethroid-resistant An. gambiae sl Covè strain which has shown over 200 fold resistance to pyrethroids mediated by a high frequency of the knockdown resistance L1014F allele (>90\%) and overexpression of the cytochrome P450 CYP6P3, associated with pyrethroid detoxification ${ }^{23}$.

\section{No evidence of cross-resistant to broflanilide and pyrethroids in CDC bottle bioassays}

Mosquito mortality rates following exposure in CDC bottles coated with alpha-cypermethrin $12.5 \mu \mathrm{g}$ were $100 \%$ with the insecticide susceptible An. gambiae sensu stricto Kisumu strain and $45 \%$ with the 
pyrethroid-resistant An. gambiae s/ Covè strain thus confirming the high levels of pyrethroid resistance in the Covè strain ${ }^{23}$. The mortality results of both strains exposed to broflanilide in the CDC bottles treated with a range of doses between $5 \mu \mathrm{g}$ and $200 \mu \mathrm{g}$ per bottle are presented in Figure 1 . Using log dosage-probit mortality analysis, the lethal concentration (LC) required to kill 50\% (LC50) and 95\% (LC95) of exposed mosquitoes were $8.5 \mu \mathrm{g}$ and $70 \mu \mathrm{g}$ respectively with the susceptible Kisumu strain and $18.1 \mu \mathrm{g}$ and $73.6 \mu \mathrm{g}$ with the pyrethroid-resistant Covè strain (Table 1). A small resistance ratio of 2.1 (95\% limits: 1.7-2.7) for the Covè strain to the Kisumu strain at the LC50 was thus detected suggesting the absence of cross-resistance to broflanilide and pyrethroids.

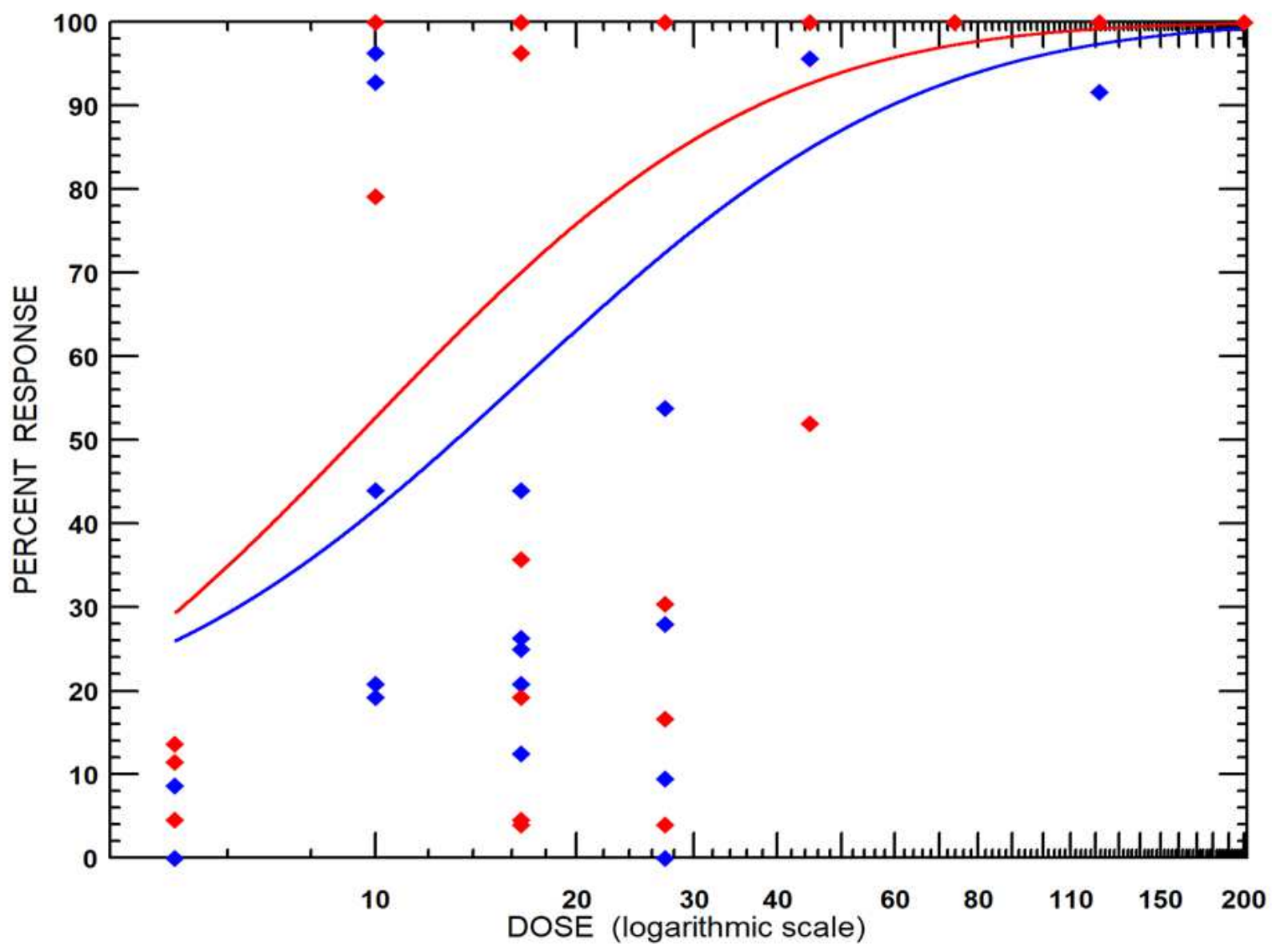

Figure 1. Mortality of susceptible An. gambiae Kisumu (red) and pyrethroid-resistant An. gambiae sl Covè (blue) mosquitoes in CDC bottle bioassays treated with a technical grade of broflanilide insecticide. Mosquitoes (150/dose) were exposed for 1-hour in cohorts of 25 per bottle and mortality recorded after $72 \mathrm{~h}$ based on preliminary findings of delayed mortality effect with broflanilide. The red line represents the response of the susceptible Kisumu strain while the blue line represents the response of the pyrethroid-resistant Covè strain. 
Table 1: Lethal dose of broflanilide on pyrethroid-resistant An. gambiae s/ Covè exposed in CDC bottle bioassays

\begin{tabular}{lcccc}
\hline Mosquito strain & Slope $(\mathrm{SE})$ & $\begin{array}{c}\text { LD50* } \\
(95 \% \mathrm{Cl})\end{array}$ & $\begin{array}{c}\text { LD95* } \\
(95 \% \mathrm{Cl})\end{array}$ & $\begin{array}{c}\text { Resistance Ratio } \\
(95 \% \mathrm{Cl})\end{array}$ \\
\hline $\begin{array}{l}\text { Susceptible } A n . \\
\text { gambiae ss Kisumu }\end{array}$ & $2.7(0.14)$ & $8.5(2.2-14.1)$ & $70(38.5-141.1)$ & - \\
\hline $\begin{array}{l}\text { Pyrethroid-resistant } \mathrm{An} . \\
\text { gambiae sl Covè }\end{array}$ & $1.8(0.20)$ & $18.1(10.7-25.4)$ & $73.6(46.9-214.9)$ & $2.1[1.7-2.7]$ \\
\hline *lethal doses are expressed in $\mu \mathrm{g} / \mathrm{ml}$ & & &
\end{tabular}

\section{Dose-response cone bioassay studies}

According to WHO guidelines ${ }^{22}$, the target dose of a new insecticide for IRS should be investigated from doses 2-4 times the minimum dose that will cause $100 \%$ mortality in a fully susceptible mosquito vector population. To identify a suitable target dose of broflanilide WP for IRS, WHO cone bioassays were conducted on cement and mud block substrates treated with a range of concentrations of broflanilide WP between $5 \mathrm{mg} / \mathrm{m}^{2}$ and $100 \mathrm{mg} / \mathrm{m}^{2}$ to detect the minimum dose that will cause $100 \%$ mortality. The cone bioassays were performed 1-week post block treatment using the insecticidesusceptible An. gambiae ss Kisumu strain. The mortality rates observed in the cone bioassays are presented in Figure 1. No knockdown was recorded with broflanilide WP at any of the doses and with any of the substrates tested. Mortality reached $100 \%$ within 24 hours at a dose of $100 \mathrm{mg} / \mathrm{m}^{2}$ on cement block substrates (Fig1a) and a dose of $12.5 \mathrm{mg} / \mathrm{m}^{2}$ on mud block substrates (Fig $1 \mathrm{~b}$ ). Broflanilide WP performed better on mud than on cement. Data with cement blocks showed a delayed mortality effect with broflanilide WP doses below $100 \mathrm{mg} / \mathrm{m}^{2}$ with mortality increasing gradually from 24 hours and reaching a peak at 72 hours. At the doses tested, there was no measurable increase in mortality when holding time was extended beyond 72 hours. This demonstrated a delayed mortality effect with broflanilide WP, as a result, for subsequent studies with the insecticide, mosquito mortality was recorded only up to 72 hours post-exposure.

A dose of $200 \mathrm{mg} / \mathrm{m}^{2}$, which was two times the dose that induced $100 \%$ mosquito mortality within 24hours on both substrates, was identified as a suitable dose for further laboratory studies on the residual efficacy of broflanilide WP. Since the insecticide performed better on mud block substrates inducing optimal mortality at even much lower doses, residual efficacy on mud block substrates was also assessed at $100 \mathrm{mg} / \mathrm{m}^{2}$. 

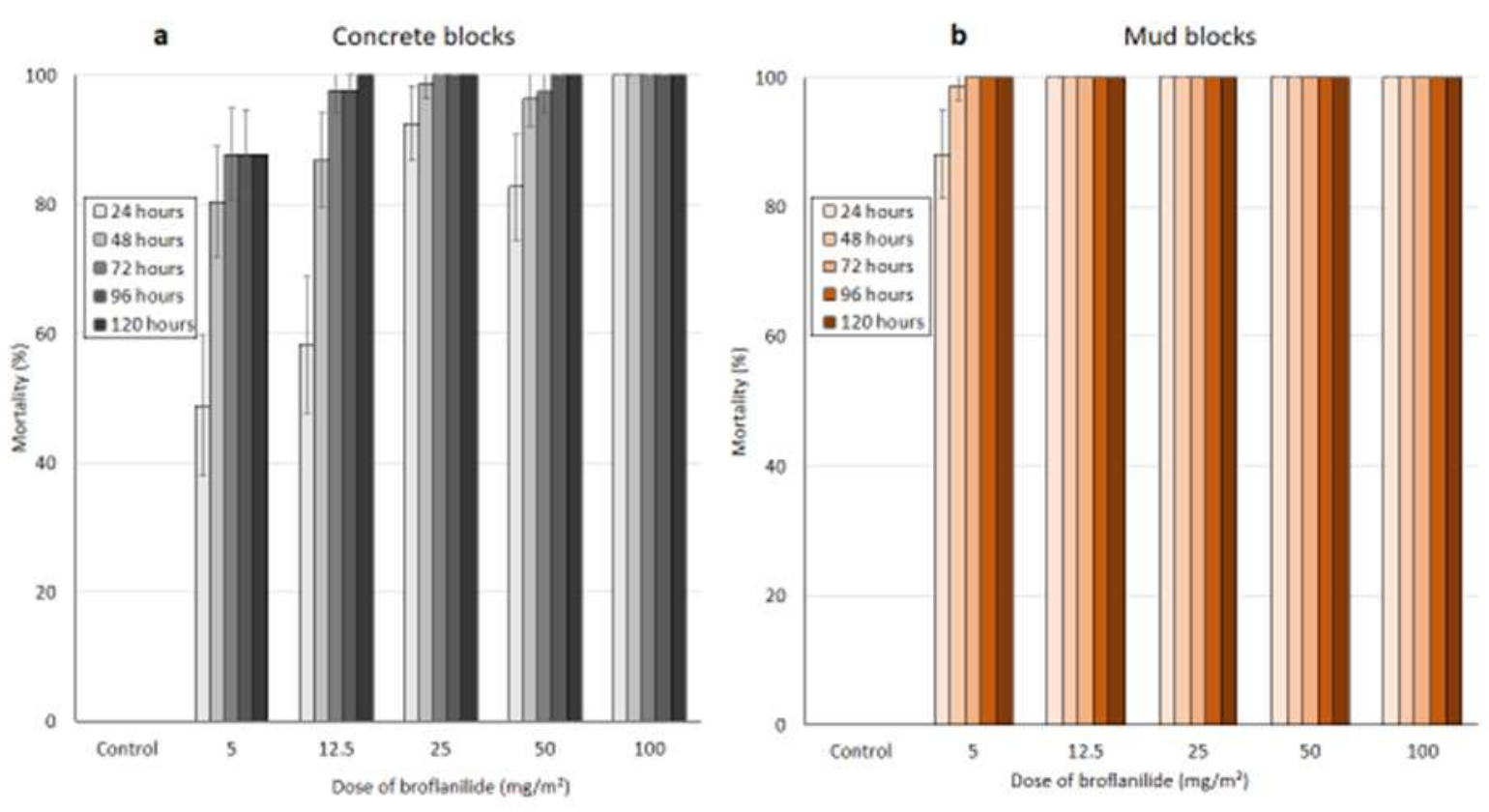

161

162

163

164

165

166

167

168

169

170

171

172

173

174

175

176

177

Figure 1: Mortality of insecticide-susceptible An. gambiae ss Kisumu strain mosquitoes on broflanilide WP treated cement (a) and mud (b) blocks substrates. Mosquitoes were exposed 1-week posttreatment for 30 -minutes in WHO cone bioassays and mortality recorded every $24 \mathrm{hrs}$ for up to $120 \mathrm{hrs}$.

\section{Broflanilide WP shows prolonged residual efficacy on block substrates in laboratory cone bioassays}

The residual efficacy of broflanilide WP was investigated at application rates of $200 \mathrm{mg} / \mathrm{m}^{2}$ on cement block substrates and $100 \mathrm{mg} / \mathrm{m}^{2}$ and $200 \mathrm{mg} / \mathrm{m}^{2}$ on mud block substrates. Blocks of each substratetype were treated at each selected dose and tested in WHO cone bioassays at 1-week post-treatment and monthly intervals subsequently using the insecticide-susceptible An. gambiae ss Kisumu and the pyrethroid-resistant An. gambiae s/ from Covè.

Monthly cone bioassay mortality $\left(72 \mathrm{~h}\right.$ ) on cement blocks treated at $200 \mathrm{mg} / \mathrm{m}^{2}$ was $>80 \%$ with the susceptible An. gambiae ss Kisumu strain for 6 months after which it ranged between $57 \%$ and $100 \%$ up to month 18 post-treatment (Fig 2). With the pyrethroid-resistant An. gambiae s/ Covè strain, mortality on cement blocks $\left(200 \mathrm{mg} / \mathrm{m}^{2}\right)$ remained $>80 \%$ for up to 14 months post-treatment (Fig 2). Monthly cone bioassay mortality of both strains at both doses tested on mud blocks $\left(100 \mathrm{mg} / \mathrm{m}^{2}\right.$ and $200 \mathrm{mg} / \mathrm{m}^{2}$ ) remained $>80 \%$ for 16 months (Fig 3 ). 


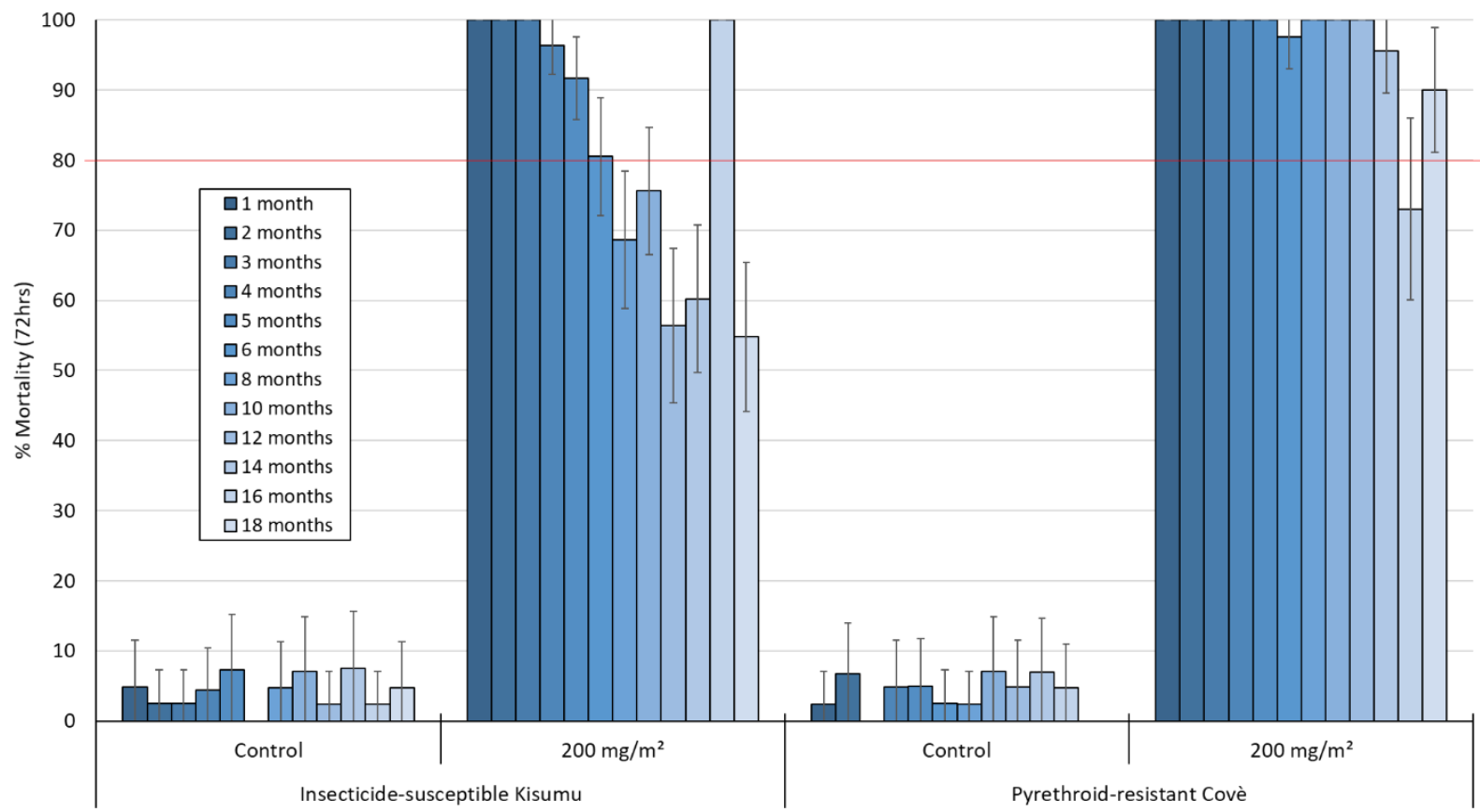

Figure 2: Monthly cone bioassays mortality of insecticide-susceptible An. gambiae ss Kisumu and pyrethroid-resistant An. gambiae s/ Covè strain mosquitoes on broflanilide WP-treated cement block substrates in the laboratory (WHO Phase 1). At each time point, forty 2-5 days old female mosquitoes were exposed for 30-minutes in WHO cone bioassays and mortality recorded after $72 \mathrm{~h}$.

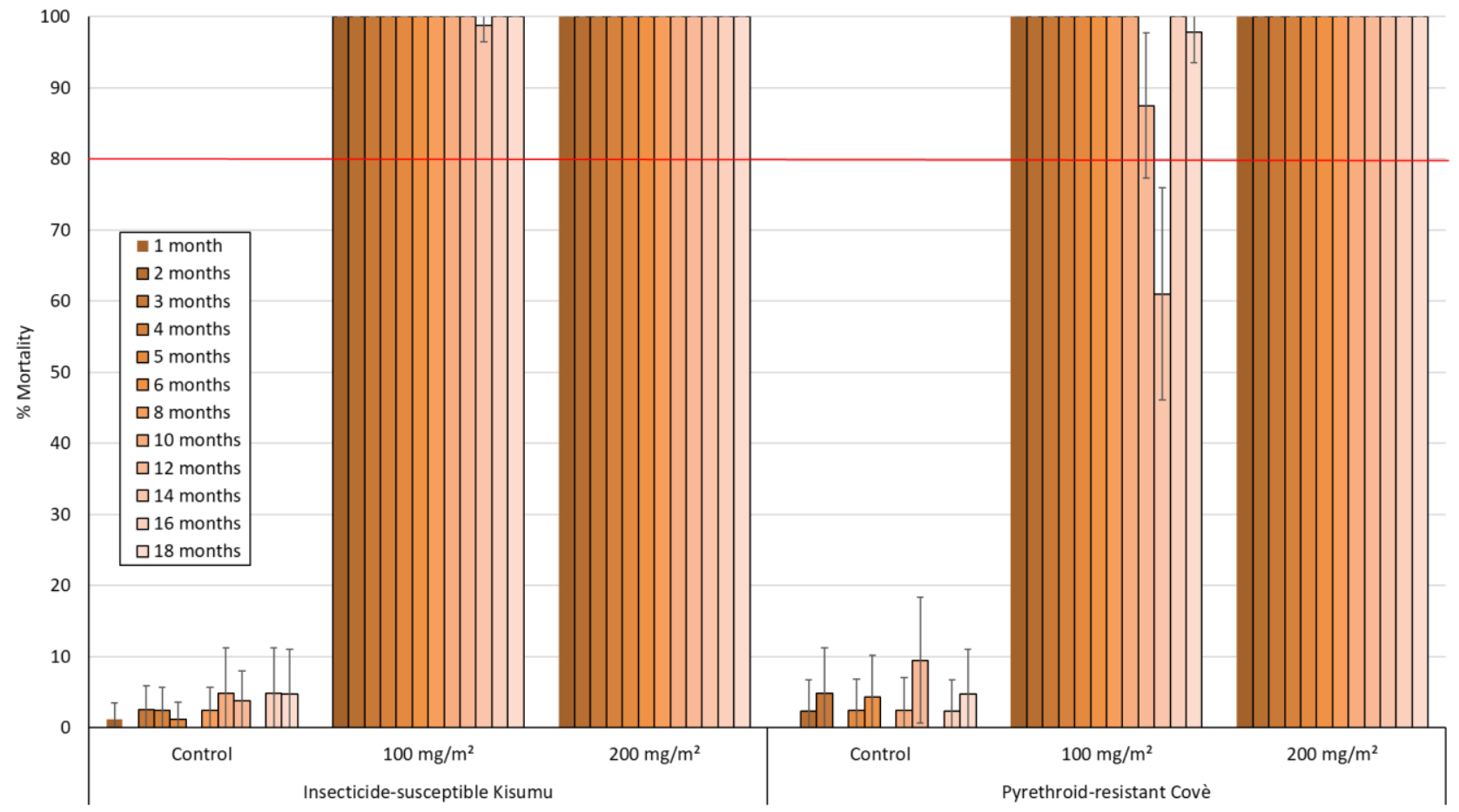

Figure 3: Monthly cone bioassays mortality of insecticide-susceptible An. gambiae ss Kisumu and pyrethroid-resistant An. gambiae sl Covè strain mosquitoes on broflanilide- treated mud block substrates. At each monthly time point, forty 2-5 days old female mosquitoes were exposed for 30minutes in WHO cone bioassays and mortality recorded after $72 \mathrm{~h}$. 
To investigate the efficacy of the broflanilide WP formulation for IRS against wild free-flying pyrethroid-resistant malaria vectors, we performed an experimental hut trial at the CREC/LSHTM experimental hut station in Covè, southern Benin $\left(7^{\circ} 14^{\prime} \mathrm{N} 2^{\circ} 18^{\prime} \mathrm{E}\right)$. The local vector population in Covè is resistant to pyrethroids and DDT. Molecular analysis has revealed a $\mathrm{kdr}$ (L1014F) allele frequency of $89 \%$ and microarray studies have also found overexpression of CYP6P3, a P450 that is as an efficient metabolizer of pyrethroids ${ }^{23}$.

Experimental huts simulate household conditions and are thus used to assess the capacity of indoor vector control interventions to prevent mosquito entry, induce early exiting of vector mosquitoes, prevent mosquito feeding and induce mosquito mortality under carefully controlled household conditions ${ }^{22,24}$. Broflanilide WP was evaluated against wild pyrethroid-resistant An gambiae sl in Covè in experimental huts treated at application rates of $100 \mathrm{mg} / \mathrm{m}^{2}$ and $150 \mathrm{mg} / \mathrm{m}^{2}$. To investigate efficacy on commonly used wall substrates in Benin, for each application rate, the inner walls and ceiling of the experimental huts were plastered with either cement or mud. Broflanilide WP was compared to primiphos-methyl CS (Actellic ${ }^{\circledR} 300 \mathrm{CS}$ ) applied at $1000 \mathrm{mg} / \mathrm{m}^{2}$ on cement walls as a positive control. At the time of this study, the toxicity and potential risk of broflanilide WP for IRS was yet to be fully assessed, so it was not acceptable to use human volunteer sleepers as hosts to attract wild vector mosquitoes into the experimental huts until a human risk-assessment was performed and the product was found safe for IRS at the potential application rates. Preliminary studies revealed that the local wild An. gambiae sl at the Covè experimental hut station were also attracted to and blood-fed on cows, although less than to humans. Hence, cows were used as replacement hosts to attract mosquitoes into the experimental huts during the trial.

\section{Mosquito exiting rates in experimental huts}

214 A total of 745 female An. gambiae sl and 771 female An. ziemanni were collected in the experimental 215 huts over the 6-month trial (Tables 2 and 3). Molecular species analysis (SINE PCR) performed using 216 the protocol proposed by Santolamazza et al ${ }^{25}$ on DNA extracted from a random selection of 100 live and dead An. gambiae s/ collected in the experimental huts during the 6-month trial, revealed that the vector was composed of $89 \%$ An. coluzzii and $11 \%$ An. gambiae ss. 
221 (95\%). Exiting rates with broflanilide WP did not also differ substantially between the two application

222 rates tested and between the two substrates assessed (Table 2).

223 A similar trend in mosquito exiting was observed with the An. ziemanni collected in the experimental 224 huts except that exiting rates with broflanilide WP-treated huts did not differ significantly from that in the negative control hut $(\mathrm{P}<0.05)$ (Table 2$)$.

Broflanilide WP induces similar overall mortality of wild pyrethroid-resistant An. gambiae s.I compared. to pirimiphos-methyl CS.

Mortality of free-flying wild, pyrethroid-resistant An. gambiae sl entering the control untreated hut was $1 \%$ while mortality with broflanilide WP treated huts ranged between $57 \%$ and $66 \%$ (Table 2 ). The mortality rates observed with broflanilide WP were similar to mortality observed with pirimiphosmethyl CS (57\% - 66\% with broflanilide WP vs. $56 \%$ with pirimiphos-methyl CS; P>0.05). For each substrate type, mortality with broflanilide WP did not differ significantly between the doses tested; cement: $57 \%$ with $100 \mathrm{mg} / \mathrm{m}^{2}$ vs $66 \%$ with $150 \mathrm{mg} / \mathrm{m}^{2}(P=0.439)$ and mud: $63 \%$ with both application rates $(P=0.922)$. Broflanilide WP generally performed the same on mud and cement substrates at both application rates; at $100 \mathrm{mg} / \mathrm{m}^{2}: 57 \%$ on cement vs. $63 \%$ on mud $(P=0.938)$ and at $150 \mathrm{mg} / \mathrm{m}^{2}$ : $66 \%$ with cement vs. $63 \%$ with mud $(P=0.664)$. In addition, for each application rate, the difference in mortality between substrates was not significant $(P>0.05)$. As in the phase 1 cone bioassays, the free-flying mosquito experimental hut data also showed a delayed mortality effect with broflanilide WP on An. gambiae sl (Figure 4). Mortality increased steadily from $35-41 \%$ at 24 hours to $57-63 \%$ at 72 hours with the $100 \mathrm{mg} / \mathrm{m}^{2}$ application rate and from $48 \%-52 \%$ at $24 \mathrm{~h}$ hours to $63 \%-66 \%$ at 72 hours with the $150 \mathrm{mg} / \mathrm{m}^{2}$ application rate $(P<0.05)$.

Mortality rates achieved with broflanilide WP against An. ziemanni (Table 3) were generally higher than what was observed with An. gambiae sl (74\%-88\% vs. 50\%-61\%). Mortality in cement huts did not differ between the two application rates tested $\left(74 \%\right.$ with $100 \mathrm{mg} / \mathrm{m}^{2}$ vs $77 \%$ with $150 \mathrm{mg} / \mathrm{m}^{2}$ (P $>0.05$ ) but for mud-walled huts, mortality was significantly higher with the higher application rate of broflanilide WP $(\mathrm{P}<0.05)$. Mortality rates achieved with broflanilide WP on cement walls were also similar to what was observed with pirimiphos-methyl CS ( $P>0.05)$. Broflanilide WP also induced delayed mortality with An. ziemanni entering huts during the trial. 
254 As expected of IRS treatments, blood-feeding rates of both mosquito species were generally very high 255 across all huts (>90\%). For pyrethroid-resistant $A n$. gambiae $s l$, there was no significant difference in 256 blood-feeding rates between the two application rates $\left(100 \mathrm{mg} / \mathrm{m}^{2}\right.$ and $\left.150 \mathrm{mg} / \mathrm{m}^{2}\right)$ of broflanilide 257 WP for either substrate type ( $>0.05$, Table 2$)$. With An. ziemanni, while a significantly lower blood258 feeding rate was observed with the higher dose of broflanilide WP in mud-walled huts, all other 259 treatments tested gave similar blood-feeding rates irrespective of substrate type (Table 3).

261 Table 2. Results with wild, free-flying pyrethroid-resistant An. gambiae s/ entering experimental huts 262 in Covè, Benin

\begin{tabular}{|c|c|c|c|c|c|c|}
\hline \multirow{3}{*}{$\begin{array}{l}\text { Insecticide } \\
\text { treatment } \\
\text { Substrate } \\
\text { Target dose }\end{array}$} & \multirow{3}{*}{$\begin{array}{c}\begin{array}{c}\text { Untreated } \\
\text { Control }\end{array} \\
\text { Cement } \\
-\end{array}$} & \multicolumn{4}{|c|}{ Broflanilide WP } & \multirow{3}{*}{$\begin{array}{c}\text { Pirimiphos- } \\
\text { methyl CS } \\
\text { Cement } \\
1000 \mathrm{mg} / \mathrm{m}^{2}\end{array}$} \\
\hline & & \multicolumn{2}{|c|}{ Cement } & \multicolumn{2}{|c|}{ Mud } & \\
\hline & & $100 \mathrm{mg} / \mathrm{m}^{2}$ & $150 \mathrm{mg} / \mathrm{m}^{2}$ & $100 \mathrm{mg} / \mathrm{m}^{2}$ & $150 \mathrm{mg} / \mathrm{m}^{2}$ & \\
\hline $\begin{array}{l}\text { Total collected } \\
\text { Total exiting } \\
\% \text { Exiting } \\
95 \% \mathrm{Cl} \\
\end{array}$ & $\begin{array}{c}156 \\
107 \\
69^{\mathrm{a}} \\
{[61-75]} \\
\end{array}$ & $\begin{array}{c}107 \\
95 \\
89^{b d} \\
{[83-95]} \\
\end{array}$ & $\begin{array}{c}56 \\
49 \\
88^{\mathrm{bd}} \\
{[79-96]} \\
\end{array}$ & $\begin{array}{c}70 \\
64 \\
91^{\mathrm{cd}} \\
{[85-98]} \\
\end{array}$ & $\begin{array}{c}88 \\
70 \\
80^{\mathrm{ab}} \\
{[71-88]} \\
\end{array}$ & $\begin{array}{c}268 \\
254 \\
95^{c} \\
{[92-97]} \\
\end{array}$ \\
\hline $\begin{array}{l}\text { No. dead after } 72 \mathrm{~h} \\
\% \text { dead after } 72 \mathrm{~h} \\
95 \% \mathrm{Cl}\end{array}$ & $\begin{array}{c}2 \\
1^{\mathrm{a}} \\
{[0-3]}\end{array}$ & $\begin{array}{c}61 \\
57^{b} \\
{[47-66]}\end{array}$ & $\begin{array}{c}37 \\
66^{\mathrm{b}} \\
{[54-78]}\end{array}$ & $\begin{array}{c}44 \\
63^{b} \\
{[52-74]}\end{array}$ & $\begin{array}{c}55 \\
63^{b} \\
{[52-72]}\end{array}$ & $\begin{array}{c}151 \\
56^{\mathrm{b}} \\
{[50-62]}\end{array}$ \\
\hline $\begin{array}{l}\text { No blood-fed } \\
\% \text { Blood-fed } \\
95 \% \mathrm{Cl} \\
\text { Blood-feeding } \\
\text { Inhibition (\%) }\end{array}$ & $\begin{array}{c}154 \\
99^{\mathrm{a}} \\
{[97-100]} \\
-\end{array}$ & $\begin{array}{c}99 \\
93^{\mathrm{b}} \\
{[88-98]} \\
6\end{array}$ & $\begin{array}{c}52 \\
93^{\mathrm{ab}} \\
{[86-99]} \\
6\end{array}$ & $\begin{array}{c}66 \\
94^{\mathrm{ab}} \\
{[89-100]} \\
5\end{array}$ & $\begin{array}{c}81 \\
92^{\mathrm{b}} \\
{[87-98]} \\
7\end{array}$ & $\begin{array}{c}259 \\
97^{\mathrm{ab}} \\
{[95-99]} \\
2\end{array}$ \\
\hline
\end{tabular}

263 Values bearing the same letter superscript along a row are not significantly different at the 5\% level 264 (P>0.05, logistic regression) 


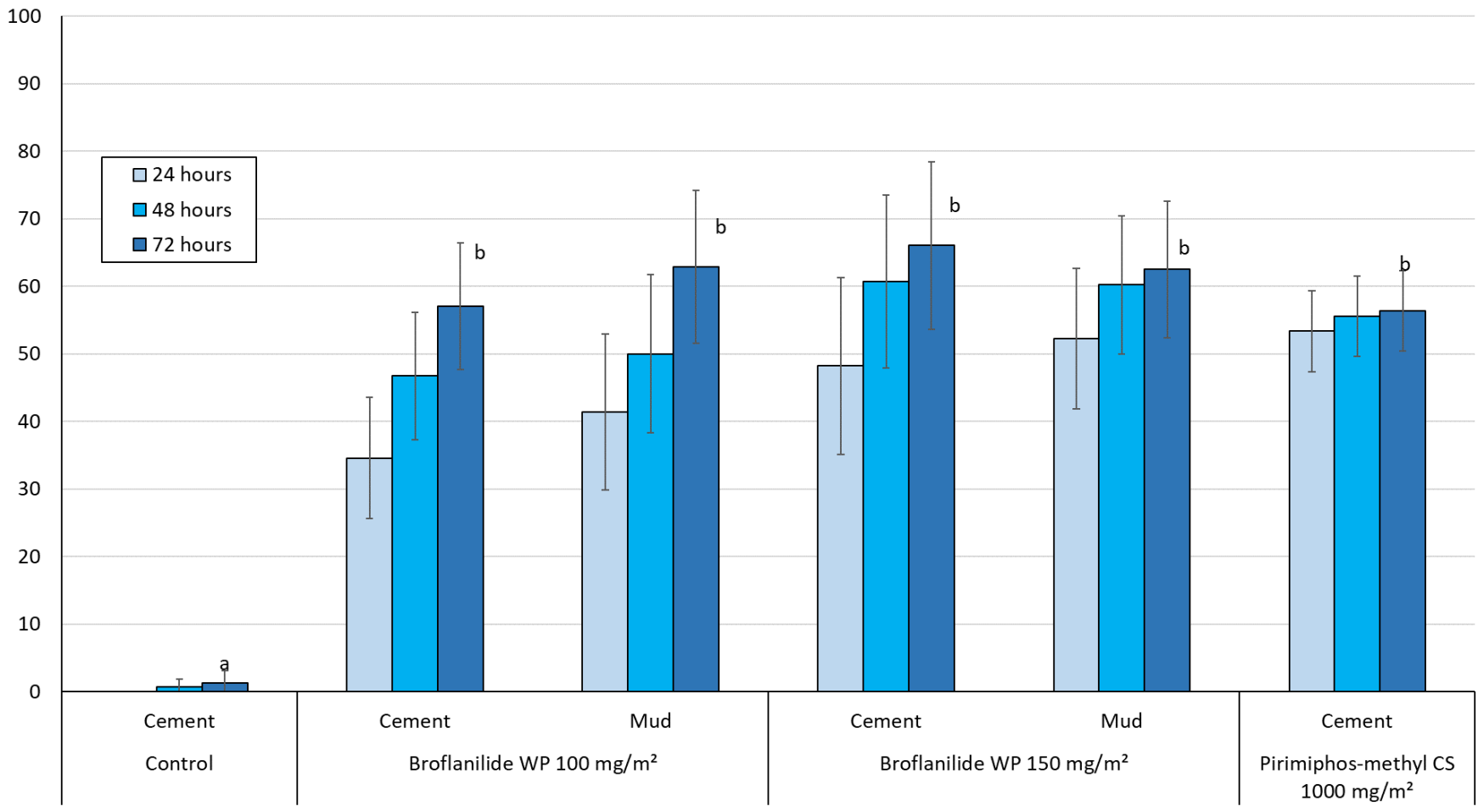

267 Figure 4: Overall mortality of wild free-flying pyrethroid-resistant An. gambiae s/ at 24, 48 and 72 268 hours after collection from experimental huts in Covè, Benin. Bars bearing the same letter label are not 269 significantly different at the 5\% level (logistic regression). Error bars represent 95\% confidence intervals. 270 Broflanilide WP induced a delayed effect on wild vector mosquitoes in experimental huts.

272 Table 3. Results with wild, free-flying pyrethroid-resistant An. ziemanni entering experimental huts in 273 Covè, Benin

\begin{tabular}{|c|c|c|c|c|c|c|}
\hline \multirow{3}{*}{$\begin{array}{l}\text { Treatment } \\
\text { Substrate } \\
\text { Target dose }\end{array}$} & \multirow{2}{*}{$\begin{array}{c}\text { Negative } \\
\text { Control } \\
\text { Cement }\end{array}$} & \multicolumn{4}{|c|}{ Broflanilide WP } & \multirow{3}{*}{$\begin{array}{c}\begin{array}{c}\text { Pirimiphos- } \\
\text { methyl CS }\end{array} \\
\text { Cement } \\
1000 \mathrm{mg} / \mathrm{m}^{2}\end{array}$} \\
\hline & & \multicolumn{2}{|c|}{ Cement } & \multicolumn{2}{|c|}{ Mud } & \\
\hline & - & $100 \mathrm{mg} / \mathrm{m}^{2}$ & $150 \mathrm{mg} / \mathrm{m}^{2}$ & $100 \mathrm{mg} / \mathrm{m}^{2}$ & $150 \mathrm{mg} / \mathrm{m}^{2}$ & \\
\hline Total collected & 92 & 181 & 123 & 67 & 132 & 166 \\
\hline Total exiting & 79 & 170 & 107 & 64 & 101 & 152 \\
\hline \% Exiting & $86^{\mathrm{ab}}$ & $94^{\mathrm{ac}}$ & $87^{\mathrm{ac}}$ & $96^{c}$ & $77^{b}$ & $92^{\mathrm{ac}}$ \\
\hline $95 \% \mathrm{Cl}$ & [79-93] & [91-97] & [81-92] & [91-100] & {$[70-84]$} & [88-96] \\
\hline No. dead after $72 \mathrm{~h}$ & 4 & 136 & 95 & 49 & 115 & 128 \\
\hline$\%$ dead after $72 \mathrm{~h}$ & $4^{\mathrm{a}}$ & $75^{b}$ & $77^{\mathrm{bc}}$ & $73^{b}$ & $87^{c}$ & $77^{b}$ \\
\hline $95 \% \mathrm{Cl}$ & {$[0-8]$} & [67-81] & [70-85] & [62-83] & [82-93] & [70-83] \\
\hline No. Blood-fed & 92 & 171 & 112 & 67 & 112 & 162 \\
\hline Blood-feeding \% & $100^{a}$ & $94^{\mathrm{a}}$ & $91^{\mathrm{a}}$ & $100^{a}$ & $85^{b}$ & $98^{a}$ \\
\hline $95 \% \mathrm{Cl}$ & [95-100] & [91-98] & [86-96] & [95-100] & [79-91] & [95-100] \\
\hline $\begin{array}{l}\text { Blood-feeding Inhibition } \\
(\%)\end{array}$ & - & 6 & 9 & - & 15 & 2 \\
\hline
\end{tabular}

274 Values bearing the same letter superscript along a row are not significantly different at the 5\% level $275 \quad(P>0.05)$ 

high and prolonged, lasting over 6 months

To assess the residual efficacy on the different hut wall substrates (mud and cement), monthly 30-min cone bioassays were performed using unfed pyrethroid-resistant An. gambiae s/ Covè and insecticidesusceptible An. gambiae ss Kisumu strains. The results from the wall cone bioassays are presented in Figures 5 and 6. Broflanilide WP performed better on cement and mud wall substrates compared to Pirimiphos-methyl CS. For both strains, cone bioassay mortality with broflanilide WP remained over $80 \%$ with both doses and substrates throughout the 6-month trial while a drop below $80 \%$ was observed with pirimiphos-methyl CS within 2-4 months.

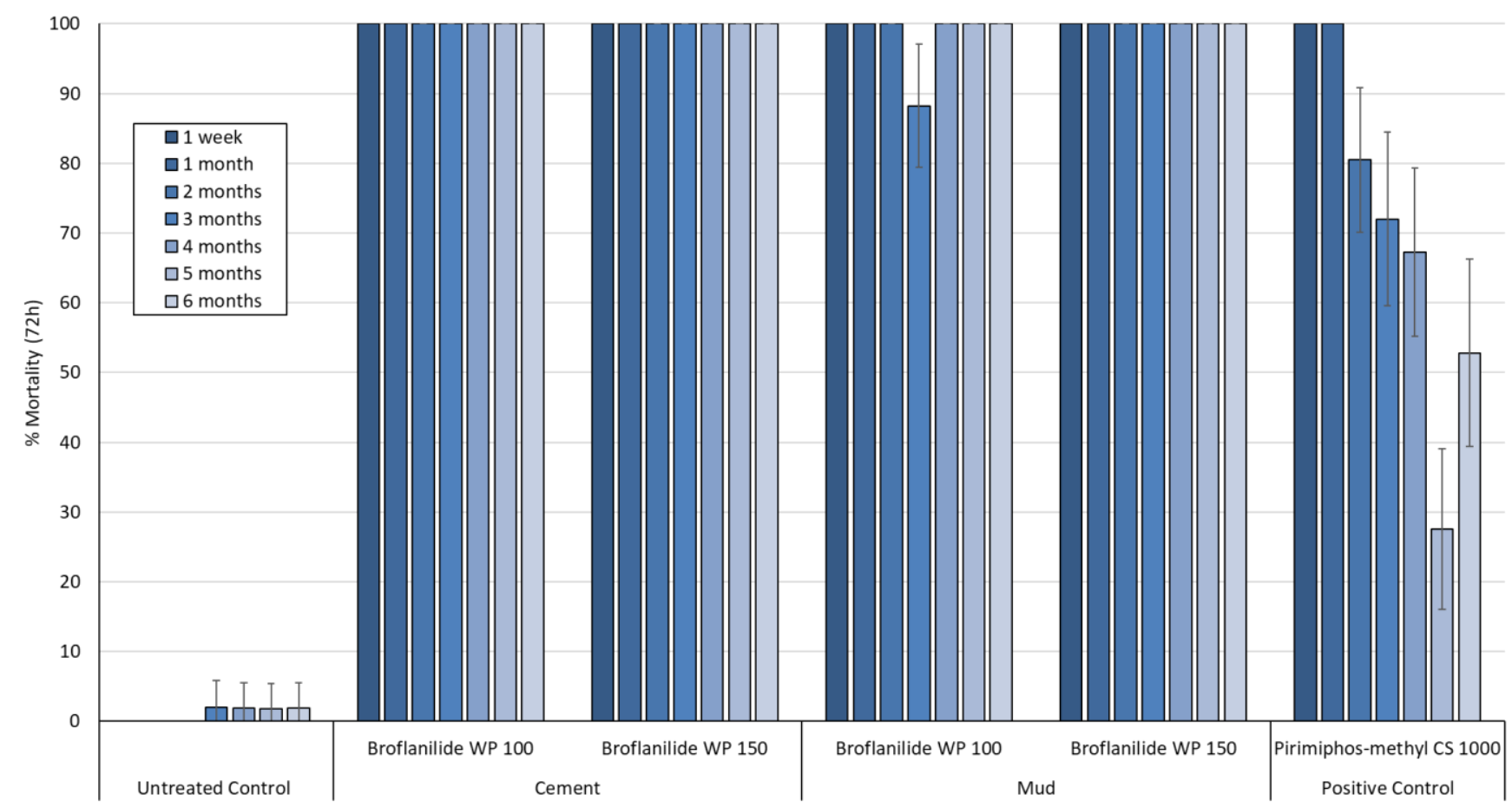

Figure 5. Cone bioassays mortality (72h) with susceptible An. gambiae ss Kisumu on broflanilide WP treated experimental hut walls. At each monthly interval, 50 2-5 days old female mosquitoes were exposed on treated of each hut in cohorts of 10 per cone. Error bars represent $95 \%$ confidence intervals. 


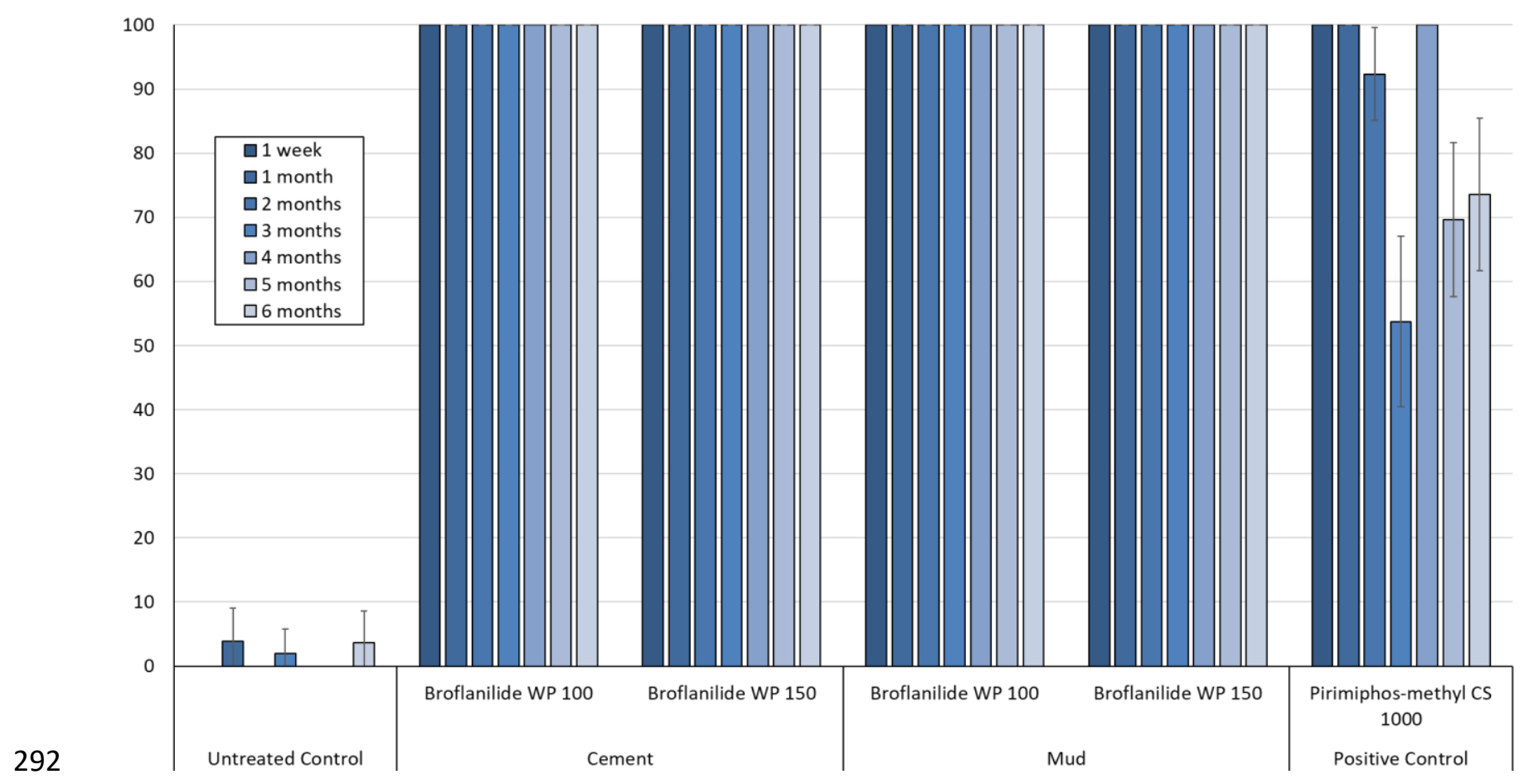

293 Figure 6. Cone bioassays mortality (72h) with pyrethroid-resistant An. gambiae s/ Covè on Broflanilide 294 WP-treated experimental hut walls. At each monthly interval, $\sim 502-5$ days old female mosquitoes 295 were exposed on treated of each hut in cohorts of 10 per cone. Error bars represent $95 \%$ confidence 296 intervals.

\section{Assessment of IRS application quality}

299 To assess the quality of the IRS applications in the experimental huts, prior to spraying, filter papers 300 measuring $5 \mathrm{~cm} \times 5 \mathrm{~cm}$ were fixed on the hut walls to be sprayed as described in the WHO guidelines $301 \quad 22$. After spraying, they were left to dry for 1 hour and then wrapped in aluminium foil and stored at $3024^{\circ} \mathrm{C}\left(+/-2^{\circ} \mathrm{C}\right)$ after which they were shipped within 2 weeks after IRS application to the Liverpool School 303 of Tropical Medicine for chemical analysis by HPLC. The summary results showed that the IRS 304 treatment applications rates were generally within an acceptable deviation of $<50 \%$ from the target 305 dose as recommended by WHO ${ }^{26}$ (Table 4).

306 Table 4. Results for chemical analysis of filter papers from experimental huts treated with 307 broflanilide WP and pirimiphos-methyl CS

\begin{tabular}{l|c|c|c|c|c|c}
\hline Treatment & $\begin{array}{c}\text { Negative } \\
\text { Control }\end{array}$ & \multicolumn{4}{|c|}{ Broflanilide WP } & Pirimiphos-methyl CS \\
\hline Substrate & Cement & \multicolumn{2}{|c|}{ Cement } & \multicolumn{2}{c}{ Mud } & Cement \\
\hline Target dose $\left(\mathrm{mg} / \mathrm{m}^{2}\right)$ & - & 100 & 150 & 100 & 150 & 1000 \\
Filter paper dosed $\left(\mathrm{mg} / \mathrm{m}^{2}\right)$ & 0 & 107 & 112 & 137 & 112 & 688 \\
$95 \% \mathrm{Cl}$ & - & {$[99-115]$} & {$[103-122]$} & {$[129-145]$} & {$[95-130]$} & {$[95-130]$} \\
Deviation from target dose & - & $+7 \%$ & $-25 \%$ & $+37 \%$ & $-19 \%$ & $-31 \%$ \\
\hline
\end{tabular}




\section{Discussion}

309

310

311

312

313

314

315

316

317

318

319

320

321

322

323

324

325

326

327

328

329

330

331

332

333

334

335

336

337

338

339

340

There is a critical need for new insecticides with novel modes of action for indoor residual spraying against malaria vectors. We investigated the bioefficacy of a newly discovered insecticide, broflanilide,

${ }^{17}$ as an indoor residual treatment in WHO phase 1 laboratory bioassays and phase 2 experimental hut studies ${ }^{22}$. Broflanilide binds to the $\gamma$-aminobutyric acid (GABA) receptor of the chloride channel at a different site to the cyclodiene insecticide dieldrin and the phenylpyrazole fipronil and thus presents a new mode of action for malaria vector control.

As a wettable powder formulation (VECTRON ${ }^{\mathrm{TM}}$ T500), broflanilide was tested for IRS against pyrethroid-susceptible and pyrethroid-resistant strains of An. gambiae sl on the principal wall substrates used in village housing in Benin. Both laboratory and experimental hut studies demonstrated the potency of this new mode of action when used for IRS. Mosquito mortality with broflanilide was high but slow compared to what is achievable with most neurotoxic public health insecticides, lasting up to 72 hours post-exposure. This delayed activity could be attributed to its mechanism of action; broflanilide has to be metabolized to its active form, desmethyl-broflanilide, before binding to its site of action ${ }^{18}$. The small resistance ratio observed in CDC bottle bioassays (2.1) and high cone bioassay mortality rates (>80\% for 6-14 months) achieved with the pyrethroid-resistant An gambiae sl strain from Covè - a strain which has shown $>200$ fold resistance to pyrethroids mediated by high kdr frequencies and overexpressed detoxifying P450 enzymes ${ }^{23}$ - would indicate the absence of cross-resistance to broflanilide and pyrethroids. This demonstrates the potential of broflanilide WP to effectively control malaria vector populations that have developed intense resistance to pyrethroids.

In the experimental hut trial against wild pyrethroid-resistant vector mosquitoes, broflanilide applied at $100 \mathrm{mg} / \mathrm{m}^{2}$ and $150 \mathrm{mg} / \mathrm{m}^{2}$ induced $57-66 \%$ mosquito mortality for 6 months. As there was no difference in wild mosquito mortality between the two application rates tested, the lower dose of $100 \mathrm{mg} / \mathrm{m}^{2}$ was chosen as a suitable IRS application rate for operational use of the insecticide in village communities.

Longer lasting IRS insecticide formulations are ideal for most endemic areas in Africa characterised by stable and extended malaria transmission as they offer continuous protection without the need for multiple resource-demanding and labour-intensive IRS campaigns. Earlier studies in Benin demonstrated the potential of the micro-encapsulated formulation of pirimiphos-methyl to provide improved and prolonged control of pyrethroid-resistant vector populations lasting 6-9 months when applied for on cement walls ${ }^{27}$. This has been followed by several reports of significantly improved malaria control with one annual IRS campaign with pirimiphos-methyl CS in many epidemiological 
settings across Africa ${ }^{28-30}$. In laboratory cone bioassays, mortality of susceptible and pyrethroidresistant vector mosquito strains with broflanilide WP remained $>80 \%$ for $6-14$ months on mud and cement block substrates. The mortality achieved against wild free-flying pyrethroid-resistant mosquitoes in the experimental huts treated with broflanilide WP was $>80 \%$ for $>6$ months demonstrating the potential of the insecticide to provide prolonged vector control and significantly improve malaria control in many endemic areas in Africa.

Although mutations in the GABA receptor conferring resistance to some non-competitive agonist agrochemicals such as cyclodienes and fipronil have been reported in malaria vectors across Africa 31,32 , the site of action of broflanilide within the GABA receptor has been demonstrated to be distinct from that of non-competitive antagonist ${ }^{20}$. This suggests a low probability of cross-resistance to broflanilide in malaria vectors. Mutations in the GABA receptor which confer resistance to metadiamides have however been detected in Drosophila ${ }^{20}$. Studies to investigate the possible presence of this mutation and other mechanisms that could confer resistance to broflanilide in wild populations of malaria vectors will be advisable.

Despite its strong anthropophilic behaviour, a considerable number of An gambiae ( $81 \%$ An. coluzzii and $19 \%$ An. gambiae ss), were attracted to the cow hosts during the experimental hut trial, although the numbers attracted were substantially lower than what would be expected in hut trials with human volunteer sleepers at the Covè experimental hut station ${ }^{14,15}$. The animal bait also attracted a more zoophilic Anopheline species into the experimental huts - An. ziemanni. Although this species has been much less studied compared to the An. gambiae complex, An. ziemanni has been previously implicated in malaria transmission in the North West region of Cameroon ${ }^{33}$ and suspected to transmit malaria in Western Kenya ${ }^{34}$. Such secondary vectors have been recognized for their importance in malaria transmission, as they may help to augment or extend the malaria transmission period. In our study, broflanilide killed $74 \%-88 \%$ of wild An. ziemanni entering the experimental huts over the 6month trial thus demonstrating the potential of the insecticide to control this Anopheline species which could be sustaining malaria transmission as a secondary vector in some parts of Africa.

Among the strategies proposed by the GPIRM for mitigating the impact of insecticide resistance in malaria vectors, the rotation of IRS formulations containing insecticides with different modes of action is currently considered the most promising strategy for insecticide resistance management ${ }^{12}$. The uptake of this strategy has been seriously limited by the very restricted number of safe and longlasting IRS insecticides available to malaria control programmes ${ }^{35}$. Two new IRS formulations containing clothianidin (a neonicotinoid) have recently been added to the WHO's list of pre-qualified 
vector control products, and are already being deployed for IRS in many endemic countries ${ }^{13}$. It is, however, crucial that vector control programmes do not become overly dependent on any one mode of action for IRS as this may lead to resistance evolving more rapidly. For the rotational strategy to work optimally, several modes of action need to be available at the same time to allow sub-national rotations and restrict selection of resistance to any single class of insecticide. Based on its novel mode of action and efficacy for IRS against pyrethroid-resistant malaria vectors as demonstrated in the present study, broflanilide WP shows potential to effectively complement other IRS formulations of insecticides in an IRS rotation plan that can manage insecticide resistance and extend the effective lives of these promising new insecticides.

\section{Conclusion}

In this study, we demonstrate for the first time the efficacy of a wettable powder formulation of broflanilide (VECTRON ${ }^{\mathrm{TM}}$ T500), a newly discovered insecticide with a novel mode of action, for IRS against malaria vectors. VECTRON ${ }^{\mathrm{TM}}$ T500 showed high activity against both pyrethroid-susceptible and resistant strains of An. gambiae s/ which lasted 6 months or more on local cement and mud substrates in both laboratory bioassays and experimental hut studies. Indoor residual spraying with broflanilide WP shows potential to provide improved and prolonged control of pyrethroid-resistant malaria vector populations.

\section{Materials and methods}

\section{WHO Phase I Laboratory bioassays}

\section{CDC bottle bioassays to investigate resistance to broflanilide}

The CDC bottle bioassays were performed with unfed 2-5 days old insecticide-susceptible An. gambiae ss Kisumu and pyrethroid-resistant An. gambiae sl adult female mosquitoes from Covè, Benin. Eight doses were selected between $5 \mu \mathrm{g} /$ bottle and $200 \mu \mathrm{g} /$ bottle as follows: $5 \mu \mathrm{g}, 10 \mu \mathrm{g}, 16.5 \mu \mathrm{g}, 27.1 \mu \mathrm{g}$, $44.7 \mu \mathrm{g}, 73.7 \mu \mathrm{g}, 121.4 \mu \mathrm{g}$ and $200 \mu \mathrm{g}$ based on results from preliminary studies. Stock solutions were prepared by serial dilutions of the technical grade insecticide in acetone. $1 \mathrm{ml}$ of each stock solution was used to coat each $250 \mathrm{ml}$ Wheaton bottle and 6 bottles were prepared per dose as described in the $C D C$ bottle bioassay guideline ${ }^{36}$. Approximately 150 female mosquitoes per insecticide dose were exposed for 1 hour in cohorts of 25 mosquitoes per bottle. Mosquitoes were also exposed to untreated control and alpha-cypermethrin $12.5 \mu \mathrm{g}$ treated bottles. Mosquitoes were held at $27^{\circ} \mathrm{C} \pm 2$ 
${ }^{\circ} \mathrm{C}$ and $80 \pm 10 \% \mathrm{RH}$ and mortality recorded after $72 \mathrm{~h}$ based on preliminary evidence of a slower mode of action of broflanilide. Estimates of the dose required to kill 50\% (LD50) and 95\% (LD95) of each strain and the resistance ratio of the wild Covè strain relative to the susceptible Kisumu strain were generated by log dosage-probit analysis (PoloPlus version 1.0).

\section{Preparation and treatment of block substrates}

Cement and mud block substrates used in cone bioassays were prepared and treated using similar methods described in previous studies by our group ${ }^{37}$. Blocks were moulded in Petri dishes $(9 \mathrm{~cm}$ diameter and $5 \mathrm{~mm}$ thick) and dried at $27{ }^{\circ} \mathrm{C} \pm 2{ }^{\circ} \mathrm{C}$ and $80 \pm 10 \% \mathrm{RH}$ for 30 days before insecticide application. Cement blocks were made by mixing cement with sand at a 1:1 ratio while mud blocks were made from local mud paste to which $10 \%$ cement was added to improve its durability and reduce cracking, in line with local practices. These substrates were treated using a Potter tower sprayer (Burkard Manufacturing Co Ltd) to achieve a homogeneous and accurate deposit of the target concentration of active ingredient (a.i) per unit area as described in WHO testing guidelines ${ }^{22}$. Blocks were weighed before and after treatments to ensure the target amount of insecticide was delivered. All treated blocks were stored, unsealed at $30^{\circ} \mathrm{C} \pm 2{ }^{\circ} \mathrm{C}, 80 \% \pm 10 \% \mathrm{RH}$ in between bioassays. Four replicate blocks of each substrate-type were prepared for each dose of insecticide tested.

\section{Dose-response cone bioassays}

The dose-response cone bioassays were performed on mud and cement blocks treated with broflanilide WP at application rates of $5 \mathrm{mg} / \mathrm{m}^{2}, 12.5 \mathrm{mg} / \mathrm{m}^{2}, 25 \mathrm{mg} / \mathrm{m}^{2}, 50 \mathrm{mg} / \mathrm{m}^{2}$ and $100 \mathrm{mg} / \mathrm{m}^{2}$, 1week post block treatment using the insecticide-susceptible An. gambiae ss Kisumu strain. For each dose and substrate-type, forty (40) unfed 2-5 days old mosquitoes were exposed for 30 minutes in cohorts of 10 mosquitoes per con and per block. Mosquitoes were held at $27^{\circ} \mathrm{C} \pm 2^{\circ} \mathrm{C}$ and $80 \% \pm 10 \%$ $\mathrm{RH}$ post-exposure and knockdown was recorded after 1 hour. To investigate delayed mosquito mortality with broflanilide WP, Mortality was recorded every 24 hours for up to 120 hours.

\section{Residual efficacy of broflanilide WP (VECTRON ${ }^{T M}$ T500) in laboratory cone bioassays}

For each insecticide dose and substrate-type used for assessment of residual efficacy, forty (40) unfed 2-5 days old female mosquitoes were exposed in cone bioassays for 30 minutes in cohorts of 10 mosquitoes per block as described in WHO guidelines ${ }^{22}$. Mosquitoes were held under the same conditions as described earlier and mortality recorded every 24 hours for up to 72 hours. Bioassays 
were performed monthly for up to 6 months post-treatment and subsequently every two months for up to 18 months post-treatment.

\section{WHO Phase II experimental hut trial}

\section{Experimental hut site}

The experimental hut site is located in an irrigated valley producing rice almost year-round and provides suitable breeding habitats for mosquitoes. The rainy season extends from March to October and the dry season from November to February. The vector population is resistant to pyrethroids and consists of both An. coluzzii and An. gambiae sensu stricto (s.s.) with the latter occurring at lower frequencies ( 23\%) and mostly in the dry season ${ }^{23}$. The experimental huts used were of the West African design and are made from cement bricks with a corrugated iron roof. Each hut was built on a cement plinth surrounded by a water-filled moat to prevent the entry of scavenging ants and had a wooden framed veranda trap to capture exiting mosquitoes ${ }^{22}$. Mosquito entry occurred via four window slits each measuring $1 \mathrm{~cm}$ and situated on three sides of the hut.

\section{Application of IRS treatments}

The following treatments were tested in 6 experimental huts:

1. Untreated hut (negative control) - cement-walled hut

2. Broflanilide WP (VECTRON ${ }^{T M}$ T500) applied at $100 \mathrm{mg} / \mathrm{m}^{2}$ - cement-walled hut

3. Broflanilide WP (VECTRON ${ }^{\mathrm{TM}}$ T500) applied at $100 \mathrm{mg} / \mathrm{m}^{2}-$ mud-walled hut

4. Broflanilide WP (VECTRON ${ }^{\top M}$ T500) applied at $150 \mathrm{mg} / \mathrm{m}^{2}$ - cement-walled hut

5. Broflanilide WP (VECTRON ${ }^{T M}$ T500) applied at $150 \mathrm{mg} / \mathrm{m}^{2}$ - mud-walled hut

6. Pirimiphos-methyl CS (Actellic ${ }^{\circledR} 300 \mathrm{CS}$ ) applied at $1000 \mathrm{mg} / \mathrm{m}^{2}$ - cement-walled hut

The IRS treatments were randomly allocated to experimental huts and were applied using a Hudson Xpert ${ }^{\circledast}$ compression sprayer equipped with a 8002 flat-fan nozzle and calibrated. To improve spray accuracy, spray swaths were marked before spraying and sprayed from the top to the bottom using the predetermined lance speed. After spraying each hut, the volume remaining in the spray tank was measured to assess the overall volume sprayed. All spray volumes were within $30 \%$ of the target. 
470 Six (6) cows were brought to sleep in the huts from 21:00 to 06:00 each trial night and were rotated between huts on successive nights to adjust for variation in individual attractiveness to mosquitoes. The cows were maintained according to institutional and national guidelines for the protection of experimental animals. The trial ran for 6 months from September 2018 to March 2019 and followed WHO guielines ${ }^{22}$. Data collection was performed for 6 nights each week. On the 7 th day of each week, the huts were cleaned and aired in preparation for the next cycle. Each morning, mosquitoes were collected from the room and veranda and brought to the laboratory where they were identified using standard identification keys and scored as fed or unfed and dead or alive. Live mosquitoes were provided with $10 \%$ glucose solution and mortality scored every 24 hours for up to 72 hours.

\section{Outcome measures in experimental huts}

The efficacy of each experimental hut treatment was expressed in terms of the following outcome measures:

- Exiting rates - the proportion of mosquitoes collected in the veranda

- Blood-feeding rates - the proportion of blood-fed mosquitoes

- Mortality - the proportion of mosquitoes found dead after a 120--hour holding time

\section{Monthly residual cone bioassays on treated experimental hut walls}

Five cones were fixed to each treated surface of each hut ( 1 per wall +1 on the ceiling). Approximately fifty (50) unfed 2-5 days old mosquitoes of each strain were exposed for 30 minutes to each hut treatment in batches of 10 mosquitoes per cone. After exposure, mosquitoes were transferred into netted plastic cups and supplied with 10\% sugar solution. Mortality was recorded every 24 hours up to 72 hours.

\section{Data management and statistical analysis}

Cone bioassay data were pooled for each substrate and dose and mean mortalities obtained using Microsoft Excel. CDC bottle bioassay data were analysed using log dosage-probit mortality analysis (Poloplus version 1.0) to determine the lethal concentration (LC) required to kill 50\% (LC50) and 95\% (LC95) of exposed mosquitoes. The diagnostic dose was defined as twice the LC95 for the insecticide- 
gambiae s.I. Covè strain was obtained by dividing its LC50 by that of the susceptible Kisumu strain. Proportional outcomes (blood-feeding, exophily and mortality) for each experimental hut treatment were analysed using blocked logistic regression in Stata version 15.1 with adjustments for the attractiveness of the individual cow hosts.

504

505

506

507

508

509

510

511

512

513

514

515

516

517

518

519

520

521

522

523

524

525

526

527

\section{Ethical considerations}

Institutional ethical approval for the study was obtained from the Ethics Review Committee of the Ministry of Health Benin (Ethical decision $n^{\circ} 39$ ). The cows used in experimental huts to attract mosquitoes were maintained following institutional standard operating procedures (SOPs) designed to improve care and protect animals used for experimentation. During the day, the cows were allowed to graze freely in an open field not too far from the experimental hut site. A veterinarian was available throughout the study to examine the cows daily and any cows found unwell was replaced and treated appropriately. All studies were performed according to relevant national and international guidelines.

12

3

(4)

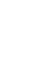

6 


\section{References}

WHO. Guidelines for malaria vector control. Geneva, Switzerland: World Health Organization (2019).

2 WHO. Indoor residual spraying : use of indoor residual spraying for scaling up global malaria control and elimination. WHO position statement. World Health Organisation https://apps.who.int/iris/handle/10665/69386 (2006).

3 Pluess, B., Tanser, F. C., Lengeler, C. \& Sharp, B. L. Indoor residual spraying for preventing malaria. Cochrane Database Syst Rev, CD006657, doi:10.1002/14651858.CD006657.pub2 (2010).

4 Cook, J. et al. Trends in parasite prevalence following 13 years of malaria interventions on Bioko island, Equatorial Guinea: 2004-2016. Malaria Journal 17, 62, doi:10.1186/s12936018-2213-9 (2018).

5 Tangena, J.-A. A. et al. Indoor residual spraying for malaria control in sub-Saharan Africa 1997 to 2017: an adjusted retrospective analysis. Malaria Journal 19, 150, doi:10.1186/s12936-020-03216-6 (2020).

6 WHO. Global report on insecticide resistance in malaria vectors: 2010-2016. Geneva, Switzerland: World Health Organization (2018).

7 Oxborough, R. M. Trends in US President's Malaria Initiative-funded indoor residual spray coverage and insecticide choice in sub-Saharan Africa (2008-2015): urgent need for affordable, long-lasting insecticides. Malar J 15, 146, doi:10.1186/s12936-016-1201-1 (2016).

8 Edi, C. V., Koudou, B. G., Jones, C. M., Weetman, D. \& Ranson, H. Multiple-insecticide resistance in Anopheles gambiae mosquitoes, Southern Cote d'Ivoire. Emerg Infect Dis 18, 1508-1511, doi:10.3201/eid1809.120262 (2012).

9 Ahoua Alou, L. P. et al. Distribution of ace-1Rand resistance to carbamates and organophosphates in Anopheles gambiae s.s. populations from Côte d'Ivoire. Malaria Journal 9, 167, doi:10.1186/1475-2875-9-167 (2010).

10 Antonio-Nkondjio, C. et al. Review of the evolution of insecticide resistance in main malaria vectors in Cameroon from 1990 to 2017. Parasit Vectors 10, 472, doi:10.1186/s13071-0172417-9 (2017).

11 Elanga-Ndille, E. et al. The G119S Acetylcholinesterase (Ace-1) Target Site Mutation Confers Carbamate Resistance in the Major Malaria Vector Anopheles gambiae from Cameroon: A Challenge for the Coming IRS Implementation. Genes (Basel) 10, doi:10.3390/genes10100790 (2019).

12 WHO. Global Plan for Insecticide Resistance Management in Malaria Vectors. . Geneva, Switzerland: World Health Organization (2012).

13 WHO. List of WHO prequalified vector control products. Geneva, Switzerland: World Health Organization https://www.who.int/pq-vector-control/prequalifiedlists/PrequalifiedProducts27January2020.pdf?ua=1 (2020).

14 Ngufor, C., Fongnikin, A., Rowland, M. \& N'Guessan, R. Indoor residual spraying with a mixture of clothianidin (a neonicotinoid insecticide) and deltamethrin provides improved control and long residual activity against pyrethroid resistant Anopheles gambiae sl in Southern Benin. PLoS One 12, e0189575, doi:10.1371/journal.pone.0189575 (2017).

15 Ngufor, C. et al. Indoor spraying with chlorfenapyr (a pyrrole insecticide) provides residual control of pyrethroid-resistant malaria vectors in southern Benin. Malaria Journal 19, 249, doi:10.1186/s12936-020-03325-2 (2020).

16 Agossa, F. R. et al. Efficacy of a novel mode of action of an indoor residual spraying product, SumiShield(R) 50WG against susceptible and resistant populations of Anopheles gambiae (s.I.) in Benin, West Africa. Parasit Vectors 11, 293, doi:10.1186/s13071-018-2869-6 (2018). 
17 Katsuta, H. et al. Discovery of broflanilide, a novel insecticide. J Pestic Sci 44, 120-128, doi:10.1584/jpestics.D18-088 (2019).

18 Nakao, T. \& Banba, S. Broflanilide: A meta-diamide insecticide with a novel mode of action. Bioorg Med Chem 24, 372-377, doi:10.1016/j.bmc.2015.08.008 (2016).

19 IRAC. The IRAC Mode of Action Classification Online. https://irac-online.org/modes-ofaction/ Accessed 24/07/2020 (2020).

20 Nakao, T., Banba, S., Nomura, M. \& Hirase, K. Meta-diamide insecticides acting on distinct sites of RDL GABA receptor from those for conventional noncompetitive antagonists. Insect Biochem Mol Biol 43, 366-375, doi:10.1016/j.ibmb.2013.02.002 (2013).

$21 \mathrm{Jia}$, Z. Q. et al. Acute toxicity, bioconcentration, elimination, action mode and detoxification metabolism of broflanilide in zebrafish, Danio rerio. J Hazard Mater 394, 122521, doi:10.1016/j.jhazmat.2020.122521 (2020).

22 WHO. Guidelines for testing mosquito adulticides for indoor residual spraying and treatment of mosquito nets. World Health Organisation Geneva, Switzerland (2006).

23 Ngufor, C. et al. Insecticide resistance profile of Anopheles gambiae from a phase II field station in Cove, southern Benin: implications for the evaluation of novel vector control products. Malar J 14, 464, doi:10.1186/s12936-015-0981-z (2015).

24 WHO. Guidelines for laboratory and field testing of long-lasting insecticidal nets. Geneva, Switzerland: World Health Organization (2013).

25 Santolamazza, F. et al. Insertion polymorphisms of SINE200 retrotransposons within speciation islands of Anopheles gambiae molecular forms. Malar J 7 (2008).

26 WHO. Data requirements and protocol for determining non-inferiority of insecticide-treated net and indoor residual spraying products within an established WHO intervention class. Geneva, Switzerland https://apps.who.int/iris/bitstream/handle/10665/276039/WHOCDS-GMP-2018.22-eng.pdf?ua=1 (2018).

27 Rowland, M. et al. A new long-lasting indoor residual formulation of the organophosphate insecticide pirimiphos methyl for prolonged control of pyrethroid-resistant mosquitoes: an experimental hut trial in Benin. PLoS One 8, e69516, doi:10.1371/journal.pone.0069516 (2013).

28 Abong'o, B. et al. Impact of indoor residual spraying with pirimiphos-methyl (Actellic 300CS) on entomological indicators of transmission and malaria case burden in Migori County, western Kenya. Scientific Reports 10, 4518, doi:10.1038/s41598-020-61350-2 (2020).

29 Salako, A. S. et al. Efficacy of Actellic 300 CS-based indoor residual spraying on key entomological indicators of malaria transmission in Alibori and Donga, two regions of northern Benin. Parasites \& Vectors 12, 612, doi:10.1186/s13071-019-3865-1 (2019).

30 Tugume, A. et al. Effects and factors associated with indoor residual spraying with Actellic 300 CS on malaria morbidity in Lira District, Northern Uganda. Malaria Journal 18, 44, doi:10.1186/s12936-019-2681-6 (2019).

31 Wondji, C. S. et al. Identification and distribution of a GABA receptor mutation conferring dieldrin resistance in the malaria vector Anopheles funestus in Africa. Insect Biochem Mol Biol 41, 484-491, doi:10.1016/j.ibmb.2011.03.012 (2011).

$32 \mathrm{Du}, \mathrm{W}$. et al. Independent mutations in the Rdl locus confer dieldrin resistance to Anopheles gambiae and An. arabiensis. Insect Mol Biol 14, 179-183, doi:10.1111/j.13652583.2005.00544.x (2005).

33 Tabue, R. N. et al. Anopheles ziemanni a locally important malaria vector in Ndop health district, north west region of Cameroon. Parasites \& Vectors 7, 262, doi:10.1186/1756-33057-262 (2014).

34 Kamau, L., Mulaya, N. \& Vulule, J. M. Evaluation of potential role of Anopheles ziemanni in malaria transmission in western Kenya. J Med Entomol 43, 774-776, doi:10.1603/00222585(2006)43[774:eoproa]2.0.co;2 (2006). 
35 Mnzava, A. P. et al. Implementation of the global plan for insecticide resistance management in malaria vectors: progress, challenges and the way forward. Malar J 14, 173, doi:10.1186/s12936-015-0693-4 (2015).

36 Brogdon W \& Chan A. Guideline for evaluating insecticide resistance in vectors using the CDC bottle bioassay. . USA: CDC Atlanta (2010).

37 Fongnikin, A. et al. Efficacy of Fludora ${ }^{\circledR}$ Fusion (a mixture of deltamethrin and clothianidin) for indoor residual spraying against pyrethroid-resistant malaria vectors: laboratory and experimental hut evaluation. Parasites \& Vectors 13, 466, doi:10.1186/s13071-020-04341-6 (2020).

\section{Declarations}

\section{Consent for publication}

\section{Not applicable}

\section{Availability of data and material}

The datasets used and/or analysed during the current study are available from the corresponding author on reasonable request.

\section{Competing interests}

The authors declare that they have no competing interests

\section{Funding}

The study was funded by the Innovative Vector Control Consortium (IVCC). The funders had no role in study design, data collection and analysis, decision to publish, or preparation of the manuscript.

\section{Authors' contributions}

$\mathrm{CN}$ co-designed the study, supervised the project, analysed the data and prepared the final manuscript. RG supervised the hut trial and contributed to data analysis and manuscript preparation. EV performed the laboratory bioassays while AF performed the hut trial. TS contributed to data analysis. MA provided administrative and logistics support. MR co-designed the study and contributed to manuscript preparation.

\section{Acknowledgements}

We thank Dr. Kunizo Mori of Mitsui Chemicals Agro, Inc for supplying the insecticide. We also thank the technical staff of CREC (Abibath Odjo, Damien Todjinou, Josias Fagbohoun, Martial Gbegbo etc) for their assistance. We appreciate Dr. Graham Small, Dr. Derric Nimmo and Dr. Sarah Rees of IVCC for coordination. We acknowledge Dr. Mark Paine of LSTM for performing the chemical analysis. We are grateful to the rice farmers at Cove for their support in the hut study.

\section{Authors' information}

Not applicable

\section{List of abbreviations}

IRS: Indoor Residual Spraying 
670 WHO: World Health Organization

671 PQ: Prequalification team

672 GPIRM: Global Plan for Insecticide Resistance Management

673 WHOPES: WHO Pesticide Evaluation Scheme

674 CREC: Centre de Recherche Entomologique de Cotonou

675 LSHTM: London School of Hygiene \& Tropical Medicine

676 Kdr: Knockdown resistance

677 


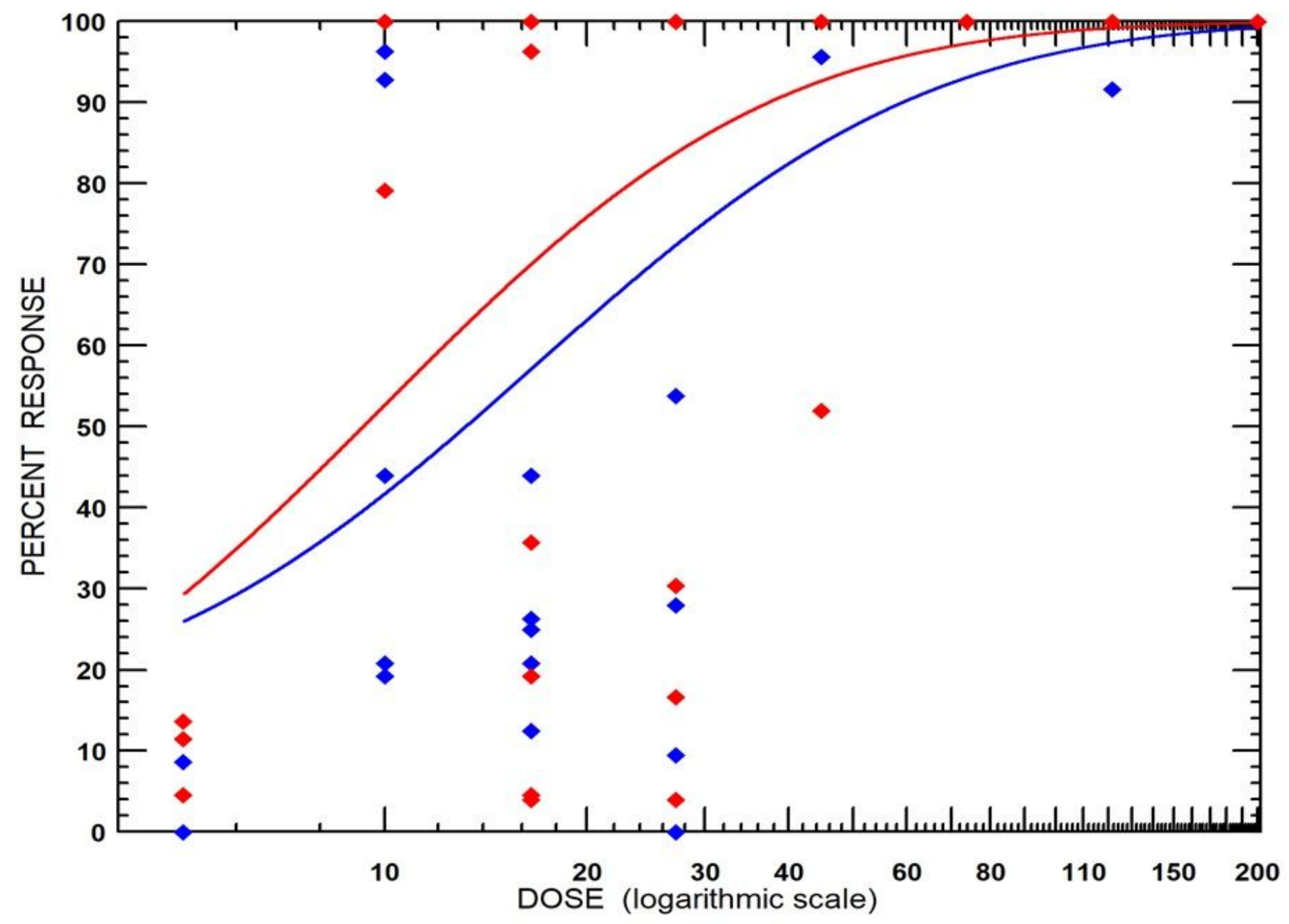

\section{Figure 1}

Mortality of susceptible An. gambiae Kisumu (red) and pyrethroid-resistant An. gambiae sl Covè (blue) mosquitoes in CDC bottle bioassays treated with a technical grade of broflanilide insecticide. Mosquitoes (150/dose) were exposed for 1-hour in cohorts of 25 per bottle and mortality recorded after $72 \mathrm{~h}$ based on preliminary findings of delayed mortality effect with broflanilide. The red line represents the response of the susceptible Kisumu strain while the blue line represents the response of the pyrethroid-resistant Covè strain. 

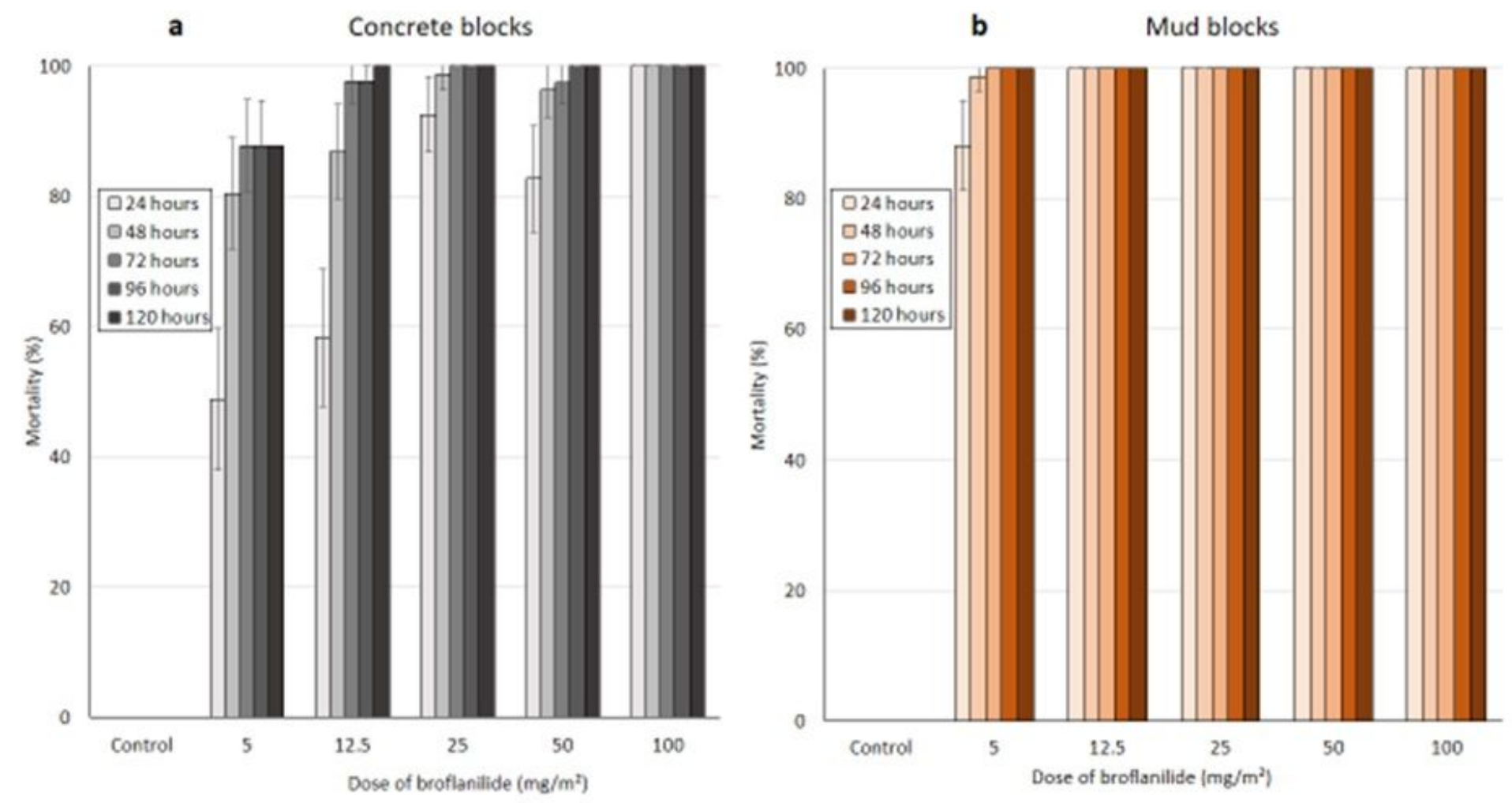

Figure 2

Mortality of insecticide-susceptible An. gambiae ss Kisumu strain mosquitoes on broflanilide WP treated cement (a) and mud (b) blocks substrates. Mosquitoes were exposed 1-week post-treatment for 30minutes in WHO cone bioassays and mortality recorded every $24 \mathrm{hrs}$ for up to $120 \mathrm{hrs}$. 


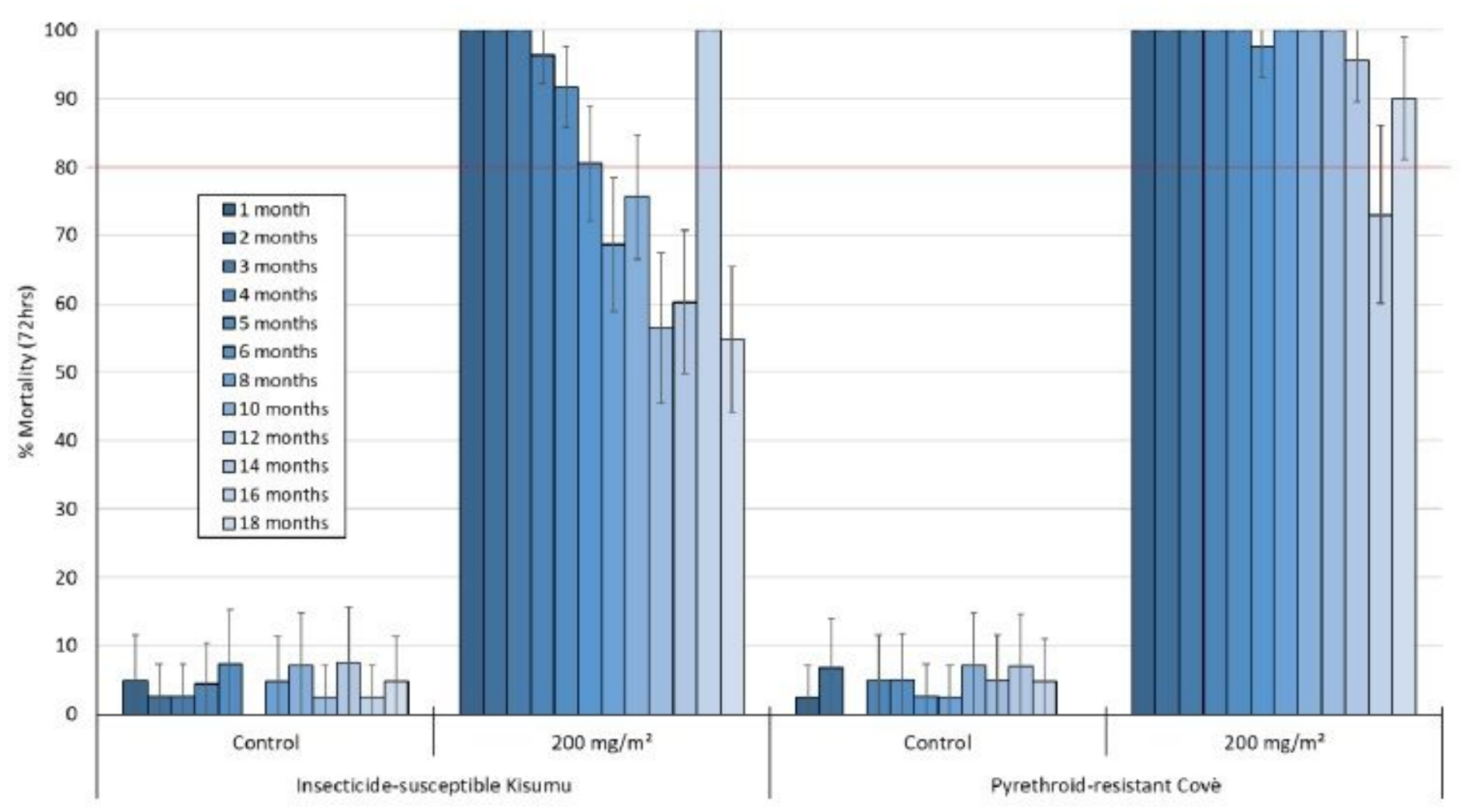

\section{Figure 3}

Monthly cone bioassays mortality of insecticide-susceptible An. gambiae ss Kisumu and pyrethroidresistant An. gambiae sl Covè strain mosquitoes on broflanilide WP-treated cement block substrates in the laboratory (WHO Phase 1). At each time point, forty 2-5 days old female mosquitoes were exposed for 30-minutes in WHO cone bioassays and mortality recorded after $72 \mathrm{~h}$. 


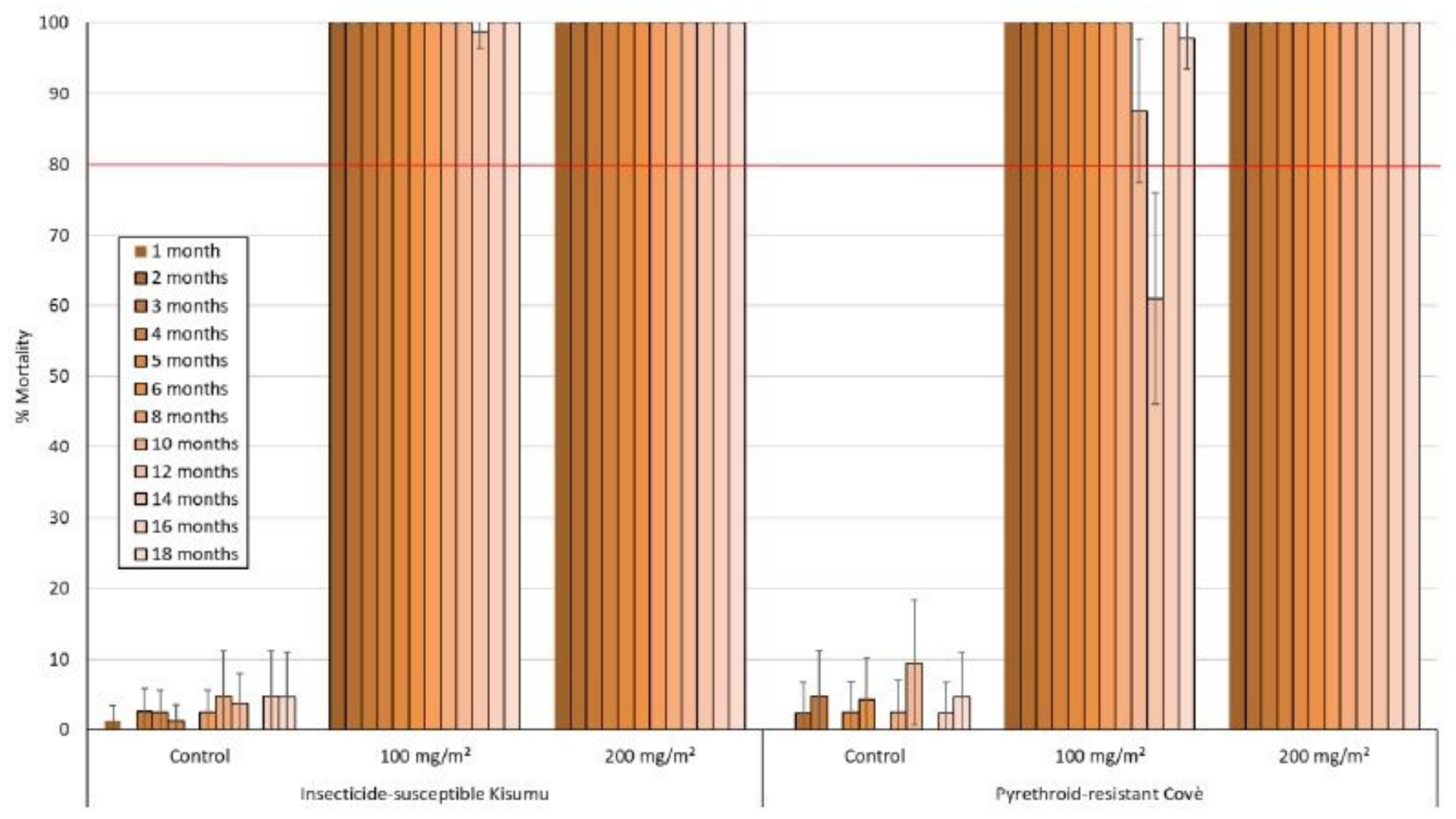

\section{Figure 4}

Monthly cone bioassays mortality of insecticide-susceptible An. gambiae ss Kisumu and pyrethroidresistant An. gambiae sl Covè strain mosquitoes on broflanilide- treated mud block substrates. At each monthly time point, forty 2-5 days old female mosquitoes were exposed for 30-minutes in WHO cone bioassays and mortality recorded after $72 \mathrm{~h}$. 


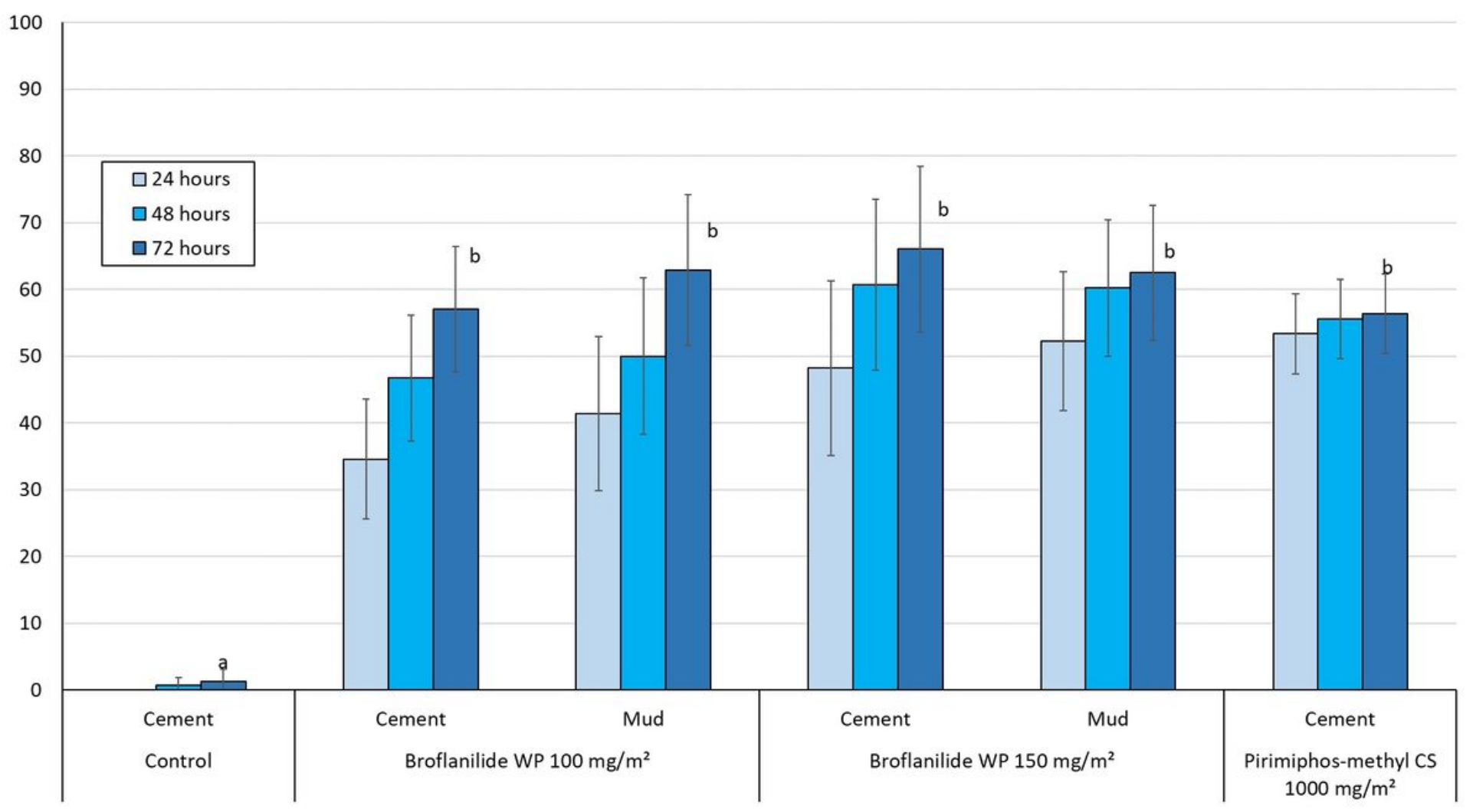

Figure 5

Overall mortality of wild free-flying pyrethroid-resistant An. gambiae sl at 24, 48 and 72 hours after collection from experimental huts in Covè, Benin. Bars bearing the same letter label are not significantly different at the $5 \%$ level (logistic regression). Error bars represent $95 \%$ confidence intervals. Broflanilide WP induced a delayed effect on wild vector mosquitoes in experimental huts. 


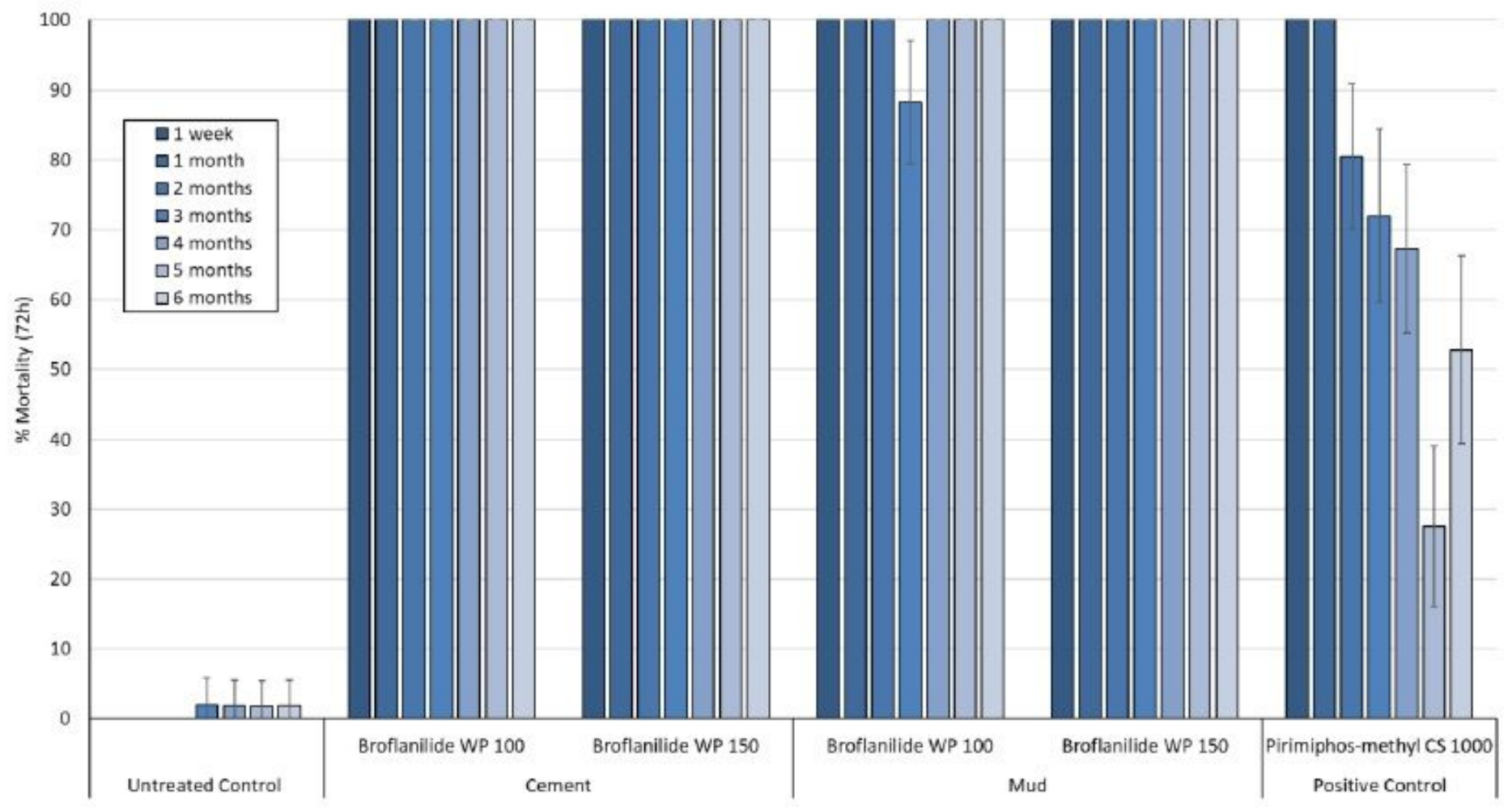

Figure 6

Cone bioassays mortality (72h) with susceptible An. gambiae ss Kisumu on broflanilide WP treated experimental hut walls. At each monthly interval, $~ 50$ 2-5 days old female mosquitoes were exposed on treated of each hut in cohorts of 10 per cone. Error bars represent $95 \%$ confidence intervals.

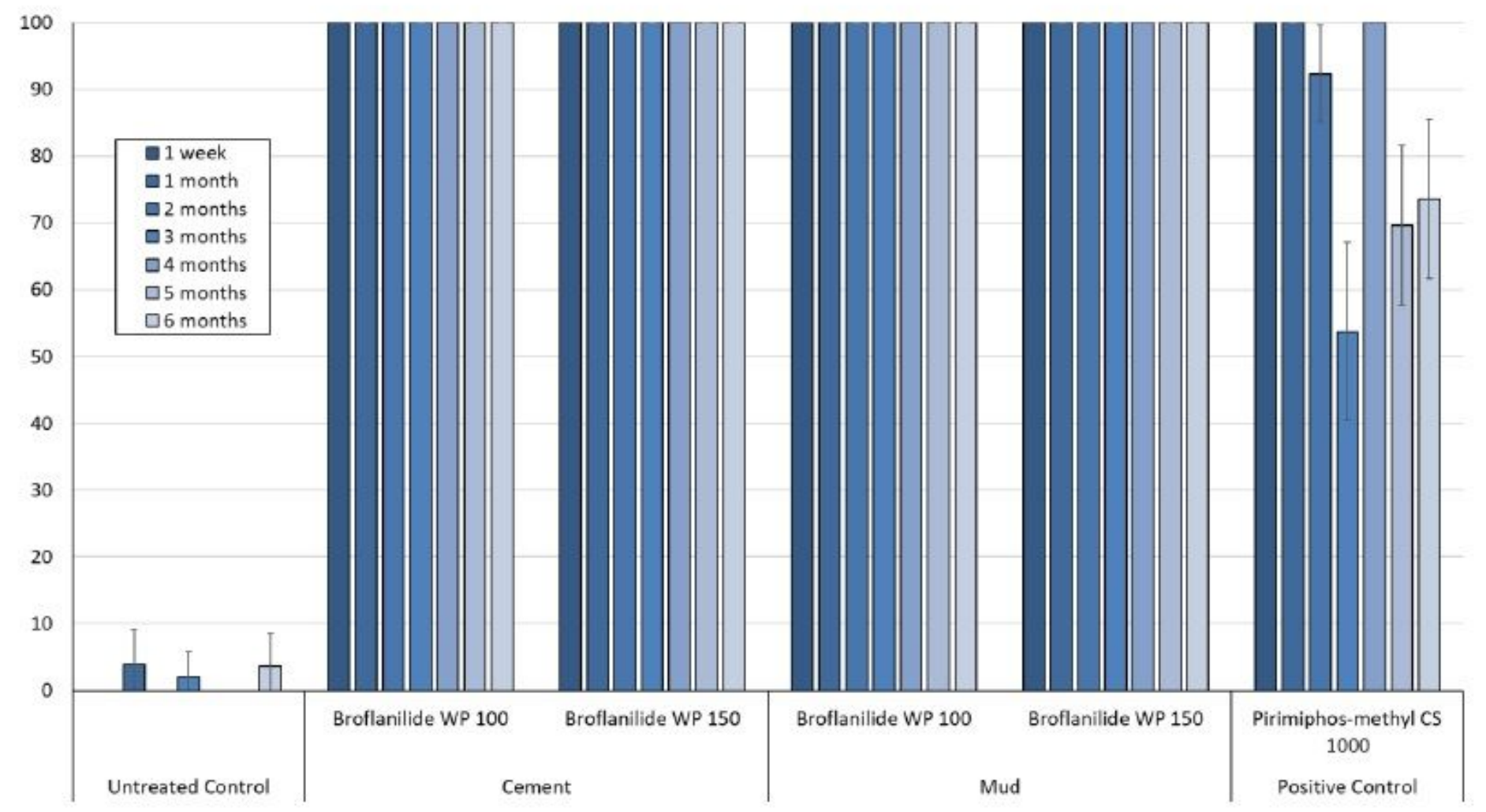




\section{Figure 7}

Cone bioassays mortality (72h) with pyrethroid-resistant An. gambiae sl Covè on Broflanilide WP-treated experimental hut walls. At each monthly interval, $~ 50$ 2-5 days old female mosquitoes were exposed on treated of each hut in cohorts of 10 per cone. Error bars represent $95 \%$ confidence intervals. 